\title{
Localized concentration of semi-classical states for nonlinear Dirac equations
}

\author{
Yanheng Ding*, Tian $\mathrm{Xu}^{\dagger}$ \\ Institute of Mathematics, Academy of Mathematics and Systems Science, \\ Chinese Academy of Sciences, 100190 Beijing, China
}

\begin{abstract}
The present paper studies concentration phenomena of semiclassical approximation of a massive Dirac equation with general nonlinear self-coupling:

$$
-i \hbar \alpha \cdot \nabla w+a \beta w+V(x) w=g(|w|) w .
$$

Compared with some existing issues, the most interesting results obtained here are twofold: the solutions concentrating around local minima of the external potential; and the nonlinearities assumed to be either super-linear or asymptotically linear at the infinity. As a consequence one sees that, if there are $k$ bounded domains $\Lambda_{j} \subset \mathbb{R}^{3}$ such that $-a<\min _{\Lambda_{j}} V=V\left(x_{j}\right)<\min _{\partial \Lambda_{j}} V, x_{j} \in \Lambda_{j}$, then the $k$-families of solutions $w_{\hbar}^{j}$ concentrates around $x_{j}$ as $\hbar \rightarrow 0$, respectively. The proof relies on variational arguments: the solutions are found as critical points of an energy functional. The Dirac operator has a continuous spectrum which is not bounded from below and above, hence the energy functional is strongly indefinite. A penalization technique is developed here to obtain the desired solutions.
\end{abstract}

Mathematics Subject Classifications (2000): $35 \mathrm{Q} 40,49 \mathrm{~J} 35$.

Keywords: Dirac equation, semi-classical states, concentration.

\section{Introduction and main result}

This paper is motivated by some works appeared in recent years concerning the nonlinear Dirac equation

$$
-i \hbar \partial_{t} \psi=i c \hbar \sum_{k=1}^{3} \alpha_{k} \partial_{k} \psi-m c^{2} \beta \psi-M(x) \psi+f(x,|\psi|) \psi
$$

\footnotetext{
*Email address: dingyh@math.ac.cn

${ }^{\dagger}$ Corresponding author, email address: xutian@amss.ac.cn
} 
for $(t, x) \in \mathbb{R} \times \mathbb{R}^{3}$. Here $c$ is the speed of light, $\hbar$ is Planck's constant, $m$ is the mass of the electron and $\alpha_{1}, \alpha_{2}, \alpha_{3}$ and $\beta$ are $4 \times 4$ complex Pauli matrices:

$$
\beta=\left(\begin{array}{cc}
I & 0 \\
0 & -I
\end{array}\right), \quad \alpha_{k}=\left(\begin{array}{cc}
0 & \sigma_{k} \\
\sigma_{k} & 0
\end{array}\right), \quad k=1,2,3,
$$

with

$$
\sigma_{1}=\left(\begin{array}{cc}
0 & 1 \\
1 & 0
\end{array}\right), \quad \sigma_{2}=\left(\begin{array}{cc}
0 & -i \\
i & 0
\end{array}\right), \quad \sigma_{3}=\left(\begin{array}{cc}
1 & 0 \\
0 & -1
\end{array}\right)
$$

The external fields $M(x)$ and $f(x,|\psi|)$ in (1.1) arise in mathematical models of particle physics, especially in nonlinear topics. They are inspired by approximate descriptions of the external forces involve only functions of fields. The nonlinear self-coupling $f(x,|\psi|)$, which describes a self-interaction in Quantum electrodynamics, gives a closer description of many particles found in the real world. Typical examples can be found in the self-interacting scalar theories, where the nonlinear function $f$ can be both polynomial and nonpolynomial (that includes the cases: $|\psi|^{\lambda}, \sin |\psi|$ etc.). Various nonlinearities are considered to be possible basis models for unified field theories (see [19], [20], [23] etc. and references therein).

Owning to the representation of (1.1), we are interested in solutions of the form $\psi(t, x)=\exp (i \xi t / \hbar) \varphi(x)$ which are called the standing waves (stationary states). It is easily checked a $\psi$ of this form satisfies equation (1.1) if and only if $\varphi$ solves

$$
-i c \hbar \sum_{k=1}^{3} \alpha_{k} \partial_{k} \varphi+m c^{2} \beta \varphi+(M(x)+\xi) \varphi=f(x,|\varphi|) \varphi .
$$

Dividing (1.2) by $c$, we are led to study the equation of the form

$$
-i \hbar \sum_{k=1}^{3} \alpha_{k} \partial_{k} \varphi+m c \beta \varphi+V(x) \varphi=g(x,|\varphi|) \varphi .
$$

Initiated by [10], the case $V(x) \equiv 0$ and $g(x,|\varphi|)=P(x)|\varphi|^{p-2}$ for $p \in(2,3)$ with a given external potential $P(x)$ is considered and a global variational technique is developed to show the least energy solution exists provided that $\hbar$ is sufficiently small and the solution concentrates around the maxima point of $P$ as $\hbar \rightarrow 0$. This method and result were later generalized jointly with co-authors in $[11,12,13]$ to competing potentials and critical nonlinearities, that is

$$
V(x) \not \equiv 0, \quad \min _{x \in \mathbb{R}^{3}} V(x)<\liminf _{|x| \rightarrow \infty} V(x),
$$


and $g(x,|\varphi|) \sim P(x)\left(|\varphi|^{p-2}+|\varphi|\right)$ for $p \in(2,3)$. As was shown in [11, $12,13]$, the semi-classical solutions concentrate around some certain points that depend on both linear and nonlinear potentials. Further investigations on the existence of solutions concentrating at certain points to nonlinear Dirac equations (including Maxwell-Dirac systems and Klein-Gordon-Dirac systems) under different conditions have also appeared in [15, 16, 17].

The method mentioned above basically depends on the global condition of the external potentials (see for example (1.4)). An interesting question, which motivates the present work, is whether one can find solutions which concentrate around local minima (or maxima) of a external potential.

As we will see, the answer is (or at least partially) affirmative. For small $\hbar$, the solitary waves are referred to as semi-classical states. The physical interpretation of such states is the following. One of the basic principles of quantum mechanics is the correspondence principle, according to which when $\hbar \rightarrow 0$, the laws of quantum mechanics must reduce to those of classical mechanics. To describe the transition from quantum to classical mechanics, the existence of solutions $\varphi_{\hbar}, \hbar$ small, possesses an important physical interest. In the present paper, denoted by $\varepsilon=\hbar, \alpha=\left(\alpha_{1}, \alpha_{2}, \alpha_{3}\right)$ and $\alpha \cdot \nabla=\sum_{k=1}^{3} \alpha_{k} \partial_{k}$, we are concerned with the following stationary nonlinear Dirac equation

$$
-i \varepsilon \alpha \cdot \nabla w+a \beta w+V(x) w=g(|w|) w, \text { for } w \in H^{1}\left(\mathbb{R}^{3}, \mathbb{C}^{4}\right)
$$

where $a=m c>0$ is constant.

Let us assume the external linear potential satisfies

$\left(V_{1}\right) V$ is locally Hölder continuous and $\max |V|<a$.

And for the nonlinear fields, by writing $G(s):=\int_{0}^{s} g(t) t d t$, we begin with the sup-linear case:

$\left(g_{1}\right) g(0)=0, g \in C^{1}(0, \infty), g^{\prime}(s)>0$

$\left(g_{2}\right) \quad(i)$ there exist $p \in(2,3), c_{1}>0$ such that $g(s) \leq c_{1}\left(1+s^{p-2}\right)$ for $s \geq 0$

(ii) there exists $\theta>2$ such that $0<G(s) \leq \frac{1}{\theta} g(s) s^{2}$ for all $s>0$;

$\left(g_{3}\right)$ the function $s \mapsto g^{\prime}(s) s+g(s)$ is nondecreasing.

Our first result is as follows

Theorem 1.1. Suppose $\left(V_{1}\right)$ and $\left(g_{1}\right)-\left(g_{3}\right)$ are satisfied. Assume additionally that there is a bounded domain $\Lambda$ in $\mathbb{R}^{3}$ such that

$$
\underline{c}:=\min _{\Lambda} V<\min _{\partial \Lambda} V .
$$

Then for all $\varepsilon>0$ small, 
(i) a solution $w_{\varepsilon} \in \cap_{q \geq 2} W^{1, q}\left(\mathbb{R}^{3}, \mathbb{C}^{4}\right)$ to (1.5) exists;

(ii) $\left|w_{\varepsilon}\right|$ possesses a (global) maximum point $x_{\varepsilon}$ in $\Lambda$ such that

$$
\lim _{\varepsilon \rightarrow 0} V\left(x_{\varepsilon}\right)=\underline{c},
$$

and

$$
\left|w_{\varepsilon}(x)\right| \leq C \exp \left(-\frac{c}{\varepsilon}\left|x-x_{\varepsilon}\right|\right)
$$

for certain constants $C, c>0$;

(iii) setting $v_{\varepsilon}(x)=w_{\varepsilon}\left(\varepsilon x+x_{\varepsilon}\right)$, as $\varepsilon \rightarrow 0$, we have $v_{\varepsilon}$ converges in $H^{1}$ to a least energy solution of

$$
-i \alpha \cdot \nabla v+a \beta v+\underline{c} v=g(|v|) v .
$$

We mention that if (1.4) is assumed, then (1.6) is satisfied naturally. So Theorem 1.1 can be seen as a local (or even a stronger) version of the consequences proved in [12].

Our next result is concerned with the asymptotically linear case. Denoted by $\widehat{G}(s):=\frac{1}{2} g(s) s^{2}-G(s)$, the hypothesis $\left(g_{2}\right)$ will be replaced by

$\left(g_{2}^{\prime}\right) \quad(i)$ there exists $b>\max |V|+a$ such that $g(s) \rightarrow b$ as $s \rightarrow \infty$;

(ii) $\widehat{G}(s)>0$ if $s>0$, and $\widehat{G}(s) \rightarrow \infty$ as $s \rightarrow \infty$.

Our result reads as

Theorem 1.2. Suppose $\left(V_{1}\right),\left(g_{1}\right),\left(g_{2}^{\prime}\right)$ and $\left(g_{3}\right)$ are satisfied. Let (1.6) be satisfied for some bounded domain $\Lambda \subset \mathbb{R}^{3}$. Then for all $\varepsilon>0$ small,

(i) a solution $w_{\varepsilon} \in \cap_{q \geq 2} W^{1, q}\left(\mathbb{R}^{3}, \mathbb{C}^{4}\right)$ to (1.5) exists;

(ii) $\left|w_{\varepsilon}\right|$ possesses a (global) maximum point $x_{\varepsilon}$ in $\Lambda$ such that

$$
\lim _{\varepsilon \rightarrow 0} V\left(x_{\varepsilon}\right)=\underline{c}
$$

and

$$
\left|w_{\varepsilon}(x)\right| \leq C \exp \left(-\frac{c}{\varepsilon}\left|x-x_{\varepsilon}\right|\right)
$$

for certain constants $C, c>0$;

(iii) setting $v_{\varepsilon}(x)=w_{\varepsilon}\left(\varepsilon x+x_{\varepsilon}\right)$, as $\varepsilon \rightarrow 0$, we have $v_{\varepsilon}$ converges in $H^{1}$ to a least energy solution of

$$
-i \alpha \cdot \nabla v+a \beta v+\underline{c} v=g(|v|) v .
$$


To our knowledge Theorem 1.2 is the first concentrating result concerned with asymptotically linear Dirac equation. Moreover, as mentioned before, our assumption (1.6) is rather weak: no restriction on the global behavior of $V$ is required other than $\left(V_{1}\right)$. In particular, the behavior of $V$ outside $\Lambda$ is irrelevant. On the other hand, hypotheses $\left(g_{1}\right)-\left(g_{3}\right)$ are satisfied by a large class of nonlinearities, which may appear in the self-interacting scalar theories, including:

1. $G(s)=s^{p}$ with $p \in(2,3)$ for the super-linear case;

2. $G(s)=\frac{b}{2} s^{2}\left(1-\frac{1}{\ln (e+s)}\right)$ for the asymptotically linear case.

Due to the above observations, we have an immediate consequence of our main theorems:

Corollary 1.3. Suppose $\left(V_{1}\right),\left(g_{1}\right),\left(g_{3}\right)$ and either $\left(g_{2}\right)$ or $\left(g_{2}^{\prime}\right)$ are satisfied. If there exist mutually disjoint bounded domains $\Lambda_{j} j=1, \ldots, k$ and constants $c_{1}<c_{2}<\cdots<c_{k}$ such that

$$
c_{j}:=\min _{x \in \Lambda_{j}} V(x)<\min _{x \in \partial \Lambda_{j}} V(x) .
$$

Then for all $\varepsilon>0$ small,

(i) there exist at least $k$ solutions $w_{\varepsilon}^{j} \in \cap_{q \geq 2} W^{1, q}\left(\mathbb{R}^{3}, \mathbb{C}^{4}\right)$ to $(1.5), j=$ $1, \ldots, k$;

(ii) $\left|w_{\varepsilon}^{j}\right|$ possesses a (global) maximum point $x_{\varepsilon}^{j}$ in $\Lambda_{j}$ such that

$$
\lim _{\varepsilon \rightarrow 0} V\left(x_{\varepsilon}^{j}\right)=c_{j}
$$

and

$$
\left|w_{\varepsilon}^{j}(x)\right| \leq C \exp \left(-\frac{c}{\varepsilon}\left|x-x_{\varepsilon}^{j}\right|\right)
$$

for certain constants $C, c>0$;

(iii) setting $v_{\varepsilon}^{j}(x)=w_{\varepsilon}^{j}\left(\varepsilon x+x_{\varepsilon}^{j}\right)$, as $\varepsilon \rightarrow 0$, we have $v_{\varepsilon}^{j}$ converges in $H^{1}$ to a least energy solution of

$$
-i \alpha \cdot \nabla v+a \beta v+c_{j} v=g(|v|) v .
$$

We remark here that in Corollary 1.3 the solutions can be separated provided $\varepsilon$ is small since $\Lambda_{j}$ are mutually disjoint. Furthermore, if $c_{1}$ in (1.7) is a global minimum of $V$, then Corollary 1.3 describes a multiple concentrating phenomenon generalizing the results in [12].

It is standard that (1.5) is equivalent to, by letting $u(x)=w(\varepsilon x)$,

$$
-i \alpha \cdot \nabla u+a \beta u+V_{\varepsilon}(x) u=g(|u|) u
$$


where $V_{\varepsilon}(x)=V(\varepsilon x)$. We will in the sequel focus on this equivalent problem. The proofs of Theorem 1.1 and Theorem 1.2 are variational, and rely upon a reduction argument and a linking structure of the energy functional associate to (1.8). Since the functional is strongly indefinite, we have to recover a compactness condition at some minimax level when $\varepsilon$ is small. All we do is to build a modification of the energy functional. In such a way, the functional is proved to satisfy the so-called Cerami compactness condition. And then, for $\varepsilon$ sufficiently small, a solution associate to the linking level is indeed a solution to the original equation. The modification of the functional corresponds to a penalization technique "outside $\Lambda$ ", and this is why no other global assumptions are required for $V$.

There have been enormous investigations on existence and concentration phenomenon of semi-classical states of nonlinear Schrödinger equation arising in the non-relativistic quantum mechanics:

$$
\hbar^{2} \Delta w-V(x) w+f(w)=0 \quad w \in H^{1}\left(\mathbb{R}^{n}\right) .
$$

It is the first time, Floer and Weinstein, in [21], proved in the one dimensional case and for $f(w)=w^{3}$ that a single spike solution concentrating around any given non-degenerate critical point of the potential $V(x)$. Oh $[28,29]$ extended this result in higher dimension and for $f(u)=|u|^{p-1} u(1<p<$ $N+2 / N-2)$. The arguments in [21, 28, 29] are based on a LyapunovSchmidt reduction and rely on the uniqueness and non-degeneracy of the ground state solutions of the autonomous problems:

$$
-\Delta v+V\left(x_{0}\right) v=f(v) \quad v \in H^{1}\left(\mathbb{R}^{n}\right) \quad\left(x_{0} \in \mathbb{R}^{n}\right) .
$$

Subsequently, variational methods were found suitable to such issues and the existence of spike layer solutions in the semi-classical limit has been established under various conditions of $V(x)$. Particularly, initiated by Rabinowitz [31], the existence of positive solutions of (1.9) for small $\hbar>0$ is proved whenever

$$
\liminf _{|x| \rightarrow \infty} V(x)>\inf _{x \in \mathbb{R}^{n}} V(x) .
$$

And these solutions concentrate around the global minimum points of $V$ when $\hbar \rightarrow 0$, as was shown by Wang [34]. It should be pointed out that M. Del Pino and P. Felmer in [7] firstly succeeded in proving a localized version of the concentration behaviour of semi-classical solutions. In [7], assuming $\inf V=V_{0}>0$ and (1.6) for some bounded domain $\Lambda$, the authors showed the existence of a single-peak solution which concentrates around the minimum points of $V$ in $\Lambda$. Their approach depends on a penalization argument and Mountain-pass theorem. Note that, since the Schrödinger operator $-\Delta+V$ is bounded from below, techniques based on the Mountain-pass theorem are well applied to the investigation. For further related results, we refer the readers to $[2,3,4,5,8,24]$ and their references, moreover, for certain results 
on solutions with multiple spike patterns for Hamiltonian elliptic systems we refer to [26].

It is quite natural to ask if certain similar results can be obtained for nonlinear Dirac equations arising in the relativistic quantum mechanics. Mathematically, the problems in Dirac equations are difficult because they are strongly indefinite in the sense that both the negative and positive parts of the spectrum of Dirac operator are unbounded and consist of essential spectrums. To this end, instead of the "Mountain-pass structure", we need a deep insight into the linking structure of strongly indefinite functional ([30]). To illustrate this point, we will see in Section 3 how local properties of $V$ lead to an essential linking structure of the strongly indefinite energy functional.

An outline of this paper is as follows: Section 2 is devoted to define the modification of the functional needed for the proof of our main results, and prove some preliminary results. In Section 3, we prove some auxiliary results and linking structure. Theorem 1.1 and Theorem 1.2 are proved in Section 4 .

\section{The variational framework}

In the sequel, by $|\cdot|_{q}$ we denote the usual $L^{q}$-norm, and $(\cdot, \cdot)_{2}$ the usual $L^{2}$-inner product. Let $H_{0}=-i \alpha \cdot \nabla+a \beta$ denote the self-adjoint operator on $L^{2} \equiv L^{2}\left(\mathbb{R}^{3}, \mathbb{C}^{4}\right)$ with domain $\mathcal{D}\left(H_{0}\right)=H^{1} \equiv H^{1}\left(\mathbb{R}^{3}, \mathbb{C}^{4}\right)$. It is well known that $\sigma\left(H_{0}\right)=\sigma_{c}\left(H_{0}\right)=\mathbb{R} \backslash(-a, a)$ where $\sigma(\cdot)$ and $\sigma_{c}(\cdot)$ denote the spectrum and the continuous spectrum. Thus the space $L^{2}$ possesses the orthogonal decomposition:

$$
L^{2}=L^{+} \oplus L^{-}, \quad u=u^{+}+u^{-}
$$

so that $H_{0}$ is positive definite (resp. negative definite) in $L^{+}$(resp. $L^{-}$). Let $E:=\mathcal{D}\left(\left|H_{0}\right|^{1 / 2}\right)=H^{1 / 2}$ be equipped with the inner product

$$
\langle u, v\rangle=\Re\left(\left|H_{0}\right|^{1 / 2} u,\left|H_{0}\right|^{1 / 2} v\right)_{2}
$$

and the induced norm $\|u\|=\langle u, u\rangle^{1 / 2}$, where $\left|H_{0}\right|$ and $\left|H_{0}\right|^{1 / 2}$ denote respectively the absolute value of $H_{0}$ and the square root of $\left|H_{0}\right|$. Since $\sigma\left(H_{0}\right)=\mathbb{R} \backslash(-a, a)$, one has

$$
a|u|_{2}^{2} \leq\|u\|^{2} \text { for all } u \in E .
$$

Note that this norm is equivalent to the usual $H^{1 / 2}$-norm, hence $E$ embeds continuously into $L^{q}$ for all $q \in[2,3]$ and compactly into $L_{l o c}^{q}$ for all $q \in[1,3)$.

It is clear that $E$ possesses the following decomposition

$$
E=E^{+} \oplus E^{-} \text {with } E^{ \pm}=E \cap L^{ \pm},
$$

orthogonal with respect to both $(\cdot, \cdot)_{2}$ and $\langle\cdot, \cdot\rangle$ inner products. And remarkably, this decomposition of $E$ induces also a natural decomposition of $L^{q}$ for every $q \in(1,+\infty)$ : 
Proposition 2.1. Let $E^{+} \oplus E^{-}$be the decomposition of $E$ according to the positive and negative part of $\sigma\left(H_{0}\right)$. Then, set $E_{q}^{ \pm}:=E^{ \pm} \cap L^{q}$ for $q \in(1, \infty)$, there holds

$$
L^{q}=\mathrm{cl}_{q} E_{q}^{+} \oplus \mathrm{cl}_{q} E_{q}^{-}
$$

with $\mathrm{cl}_{q}$ denoting the closure in $L^{q}$. More precisely, there exists $d_{q}>0$ for every $q \in(1, \infty)$ such that

$$
d_{q}\left|u^{ \pm}\right|_{q} \leq|u|_{q} \quad \text { for all } u \in E \cap L^{q} .
$$

In $L^{q}$ 's (for $q \neq 2$ ), by $\oplus$ we mean the topologically direct sum. Before proving Proposition 2.1 we would like to introduce the following definition for Multipliers (see [32, Chapter 4]) which plays an important role in our arguments.

Definition 2.2. Let $m$ be a bounded measurable function on $\mathbb{R}^{n}$, we associate a linear operator $T_{m}$ on $L^{2} \cap L^{q}$ by $\left(T_{m} u\right)^{\wedge}(\xi)=m(\xi) \hat{u}(\xi)$ where $\hat{u}$ denotes the Fourier transform of $u$. We say that $m$ is a multiplier for $L^{q}(1 \leq q \leq \infty)$ if whenever $u \in L^{2} \cap L^{q}$ then $T_{m} u \in L^{q}$ (notice it is automatically in $L^{2}$ ), and $T_{m}$ is bounded, that is,

$$
\left|T_{m} u\right|_{q} \leq A \cdot|u|_{q}, \quad u \in L^{2} \cap L^{q} \text { (with } A \text { independent of } u \text { ). }
$$

Observe that if (2.4) is satisfied, and $p<\infty$, then $T_{m}$ has a unique bounded extension to $L^{q}$, which again satisfies the same inequality.

Proof of Proposition 2.1. First we remark that in this context, the spatial domain is $\mathbb{R}^{3}$. Now recall the definitions for the matrices $\sigma_{k}, k=1,2,3$ (see Pauli matrices), and let us study $H_{0}=-i \alpha \cdot \nabla+a \beta$. It is a differential operator with constant coefficients. In the Fourier domain $\xi=\left(\xi_{1}, \xi_{2}, \xi_{3}\right)$, it becomes the operator of multiplication by the matrix

$$
\hat{H}_{0}(\xi)=\left(\begin{array}{cc}
0 & \sum_{k=1}^{3} \xi_{k} \sigma_{k} \\
\sum_{k=1}^{3} \xi_{k} \sigma_{k} & 0
\end{array}\right)+\left(\begin{array}{cc}
a & 0 \\
0 & -a
\end{array}\right) .
$$

By classical calculus we have that $\hat{H}_{0}(\xi)$ has two eigenvalues: $\pm \sqrt{a^{2}+|\xi|^{2}}$. Now, denote $P^{ \pm}$the projections on $E$ with kernel $E^{\mp}$. We see that in the Fourier domain, $P^{ \pm}$are multiplication operators by bounded smooth matrix-valued functions of $\xi$ :

$$
\begin{gathered}
\left(P^{+} u\right)^{\wedge}(\xi)=\left(\frac{1}{2}+\frac{a}{2 \sqrt{a^{2}+|\xi|^{2}}}\right)\left(\begin{array}{cc}
I & \Sigma(\xi) \\
\Sigma(\xi) & A(\xi)
\end{array}\right)\left(\begin{array}{c}
\hat{U} \\
\hat{V}
\end{array}\right) \\
\left(P^{-} u\right)^{\wedge}(\xi)=\left(\frac{1}{2}+\frac{a}{2 \sqrt{a^{2}+|\xi|^{2}}}\right)\left(\begin{array}{cc}
A(\xi) & -\Sigma(\xi) \\
-\Sigma(\xi) & I
\end{array}\right)\left(\begin{array}{c}
\hat{U} \\
\hat{V}
\end{array}\right)
\end{gathered}
$$


with $I$ being the $2 \times 2$ identity matrix and

$$
A(\xi)=\frac{\sqrt{a^{2}+|\xi|^{2}}-a}{a+\sqrt{a^{2}+|\xi|^{2}}} \cdot I, \quad \Sigma(\xi)=\sum_{k=1}^{3} \frac{\xi_{k} \sigma_{k}}{a+\sqrt{a^{2}+|\xi|^{2}}} .
$$

Here we have used the notation $\hat{u}=(\hat{U}, \hat{V}) \in \mathbb{C}^{4}$ and $\hat{U}=\left(\hat{u}_{1}, \hat{u}_{2}\right) \in \mathbb{C}^{2}$, $\hat{V}=\left(\hat{u}_{3}, \hat{u}_{4}\right) \in \mathbb{C}^{2}$.

In order that $P^{ \pm}$are multipliers for $L^{q}$, we need to use the Marcinkiewicz multiplier theorem on $\mathbb{R}^{3}$ (see [32, Chapter 4, Theorem 6']). A direct calculation shows that, for each component, the absolute value of all $k$-th $(0<k \leq 3)$ order partial derivatives for the multiplication functions are bounded by $B /|\xi|^{k}$ for some constant $B>0$. And hence, as an immediate consequence, $P^{ \pm}$are multipliers for $L^{q}$ for all $q \in(1, \infty)$. This implies that $P^{ \pm}$are continuous with respect to the $L^{q}$-norms. By noting that $P^{ \pm}\left(E^{\mp}\right)=\{0\}$, one easily sees that $P^{ \pm}$extend to continuous projections on $L^{q}$ (still denoted by $\left.P^{ \pm}\right)$with $P^{ \pm}\left(\mathrm{cl}_{q} E_{q}^{\mp}\right)=\{0\}$. And this completes the proof.

Remark 2.3. It is of great importance for the projections from $H^{1 / 2}:=$ $E=E^{+} \oplus E^{-}$onto $E^{+}$(or $E^{-}$) to be continuous in the $L^{q}$ 's and not only in $H^{1 / 2}$. This is not the case for every direct sum in $H^{1 / 2}$. In fact, the proof of Proposition 2.1 implies on the splitting of $L^{q}$ 's that: For every $q \in(1, \infty)$, $L^{q}$ can be split into topologically direct sum of two (infinite dimensional) subspaces which, accordingly, are the positive and negative projected spaces of the Dirac operator $H_{0}$.

To introduce the variational formulation of problem (1.8), we define the "energy" functional

$$
\begin{aligned}
\Phi_{\varepsilon}(u) & =\frac{1}{2} \int_{\mathbb{R}^{3}} H_{0} u \cdot \bar{u}+\frac{1}{2} \int_{\mathbb{R}^{3}} V_{\varepsilon}(x)|u|^{2}-\int_{\mathbb{R}^{3}} G(|u|) \\
& =\frac{1}{2}\left(\left\|u^{+}\right\|^{2}-\left\|u^{-}\right\|^{2}\right)+\frac{1}{2} \int_{\mathbb{R}^{3}} V_{\varepsilon}(x)|u|^{2}-\Psi(u)
\end{aligned}
$$

for $u=u^{+}+u^{-} \in E$. Standard arguments show that, under our assumptions, $\Phi_{\varepsilon} \in C^{2}(E, \mathbb{R})$ and any critical point of $\Phi_{\varepsilon}$ is a (weak) solution to (1.8).

Next, we introduce a modification of (2.5), afterwards, we will prove the modified functional satisfies the $(C)_{c}$ condition. Choose $\xi>0$ be the value at which $g^{\prime}(\xi) \xi+g(\xi)=\frac{a-|V|_{\infty}}{2}$. Let us consider $\tilde{g} \in C^{1}(0, \infty)$ such that

$$
\frac{d}{d s}(\tilde{g}(s) s)=\left\{\begin{array}{l}
g^{\prime}(s) s+g(s) \quad \text { if } s<\xi \\
\frac{a-|V|_{\infty}}{2} \quad \text { if } s>\xi
\end{array}\right.
$$

and define

$$
f(\cdot, s)=\chi_{\Lambda} g(s)+\left(1-\chi_{\Lambda}\right) \tilde{g}(s)
$$


where $\Lambda$ is a bounded domain as in the assumptions of Theorem 1.1 and $\chi_{\Lambda}$ denotes its characteristic function. One should keep in mind here that $\Lambda$ has to be rescaled when we consider the modified rescaled equation (1.8).

It is standard to check that $\left(g_{1}\right)$ and $\left(g_{3}\right)$ implies that $f$ is a Caratheodory function and it satisfies

$\left(f_{1}\right) f_{s}(x, s)$ exists everywhere, $f(x, s) s=o(s)$ uniformly in $x$ as $s \rightarrow 0$;

$\left(f_{2}\right) 0 \leq f(x, s) s \leq g(s) s$ for all $x$

$\left(f_{3}\right) \quad 0<2 F(x, s) \leq f(x, s) s^{2} \leq \frac{a-|V|_{\infty}}{2} s^{2}$ for all $x \notin \Lambda$ and $s>0 ;$

$\left(f_{4}\right) \quad(i)$ if $\left(g_{2}\right)$ is satisfied, then $0<F(x, s) \leq \frac{1}{\theta} f(x, s) s^{2}$ for all $x \in \Lambda$ and $s>0$,

(ii) if $\left(g_{2}^{\prime}\right)$ is satisfied, then $\widehat{F}(x, s)>0$ if $s>0$;

$\left(f_{5}\right) \frac{d}{d s}(f(x, s) s) \geq 0$ for all $x$ and $s>0$;

$\left(f_{6}\right)$ either $\left(g_{2}\right)$ or $\left(g_{2}^{\prime}\right)$ is satisfied, $\widehat{F}(x, s) \rightarrow \infty$ as $s \rightarrow \infty$ uniformly in $x$.

Here we used the notation $F(x, s)=\int_{0}^{s} f(x, t) t d t$ and $\widehat{F}(x, s)=\frac{1}{2} f(x, s) s^{2}-$ $F(x, s)$. Now, we define the modified functional $\widetilde{\Phi}_{\varepsilon}: E \rightarrow \mathbb{R}$ as

$$
\widetilde{\Phi}_{\varepsilon}(u)=\frac{1}{2}\left(\left\|u^{+}\right\|^{2}-\left\|u^{-}\right\|^{2}\right)+\frac{1}{2} \int_{\mathbb{R}^{3}} V_{\varepsilon}(x)|u|^{2}-\Psi_{\varepsilon}(u),
$$

where $\Psi_{\varepsilon}(u)=\int_{\mathbb{R}^{3}} F(\varepsilon x,|u|)$. Then, we see that $\widetilde{\Phi}_{\varepsilon} \in C^{2}(E, \mathbb{R})$.

We show next $\widetilde{\Phi}_{\varepsilon}$ satisfies the compactness condition. In virtue of $\left(f_{4}\right)(i)$, we have

$$
\widehat{F}(x, s) \geq \frac{\theta-2}{2 \theta} f(x, s) s^{2} \geq \frac{\theta-2}{2} F(x, s)>0
$$

for all $x \in \Lambda$ and $s>0$ provided $\left(g_{2}\right)$ is satisfied. Recall that, by $\left(f_{1}\right)$ and $\left(f_{2}\right)$, there exist $r_{1}>0$ small enough and $a_{1}>0$ such that

$$
f(x, s) \leq \frac{a-|V|_{\infty}}{4} \text { for all } s \leq r_{1}, \quad x \in \mathbb{R}^{3}
$$

and if $\left(g_{2}\right)$ is satisfied, for $s \geq r_{1}, f(x, s) \leq a_{1} s^{p-2}$, so $(f(x, s) s)^{\sigma_{0}-1} \leq a_{2} s$ with

$$
\sigma_{0}:=\frac{p}{p-1}
$$

which, jointly with $\left(f_{4}\right)(i)$, yields (see $(2.7)$ )

$$
(f(x, s) s)^{\sigma_{0}} \leq a_{2} f(x, s) s^{2} \leq a_{3} \widehat{F}(x, s) \text { for all } s \geq r_{1} \text { and } x \in \Lambda .
$$

Lemma 2.4. For each $\varepsilon>0$, let $\left\{u_{n}\right\}$ be a sequence such that $\widetilde{\Phi}_{\varepsilon}\left(u_{n}\right)$ is bounded and $\left(1+\left\|u_{n}\right\|\right) \widetilde{\Phi}_{\varepsilon}^{\prime}\left(u_{n}\right) \rightarrow 0$. Then $\left\{u_{n}\right\}$ has a convergent subsequence. 
Proof. We first show that the sequence $\left\{u_{n}\right\}$ is bounded in $E$. In fact, the representation of $\widetilde{\Phi}_{\varepsilon}$ implies that there is $C>0$ such that

$$
C \geq \widetilde{\Phi}_{\varepsilon}\left(u_{n}\right)-\frac{1}{2} \widetilde{\Phi}_{\varepsilon}^{\prime}\left(u_{n}\right) u_{n}=\int \widehat{F}\left(\varepsilon x,\left|u_{n}\right|\right)
$$

and

$$
\begin{aligned}
o(1)= & \widetilde{\Phi}_{\varepsilon}^{\prime}\left(u_{n}\right)\left(u_{n}^{+}-u_{n}^{-}\right) \\
= & \left\|u_{n}\right\|^{2}+\Re \int V_{\varepsilon}(x) u_{n} \cdot \overline{\left(u_{n}^{+}-u_{n}^{-}\right)} \\
& -\Re \int f\left(\varepsilon x,\left|u_{n}\right|\right) u_{n} \cdot \overline{\left(u_{n}^{+}-u_{n}^{-}\right)} .
\end{aligned}
$$

Case 1. $\left(f_{4}\right)(i)$ occurs.

By the definition of $f$ and (2.11), we have

$$
\begin{aligned}
& \left\|u_{n}\right\|^{2}-|V|_{\infty} \int\left|u_{n}\right| \cdot\left|u_{n}^{+}-u_{n}^{-}\right| \\
\leq & \int f\left(\varepsilon x,\left|u_{n}\right|\right)\left|u_{n}\right| \cdot\left|u_{n}^{+}-u_{n}^{-}\right|+o(1) \\
\leq & \int_{\Lambda_{\varepsilon}} f\left(\varepsilon x,\left|u_{n}\right|\right)\left|u_{n}\right| \cdot\left|u_{n}^{+}-u_{n}^{-}\right|+\frac{a-|V|_{\infty}}{2} \int\left|u_{n}\right| \cdot\left|u_{n}^{+}-u_{n}^{-}\right| \\
& +o(1),
\end{aligned}
$$

where $\Lambda_{\varepsilon}:=\left\{x \in \mathbb{R}^{3}: \varepsilon x \in \Lambda\right\}$. Thus, from (2.8) and (2.9), we easily check that

$$
\begin{aligned}
& \frac{a-|V|_{\infty}}{4 a}\left\|u_{n}\right\|^{2} \\
\leq & \int_{\left\{x \in \Lambda_{\varepsilon}:\left|u_{n}(x)\right| \geq r_{1}\right\}} f\left(\varepsilon x,\left|u_{n}\right|\right)\left|u_{n}\right| \cdot\left|u_{n}^{+}-u_{n}^{-}\right|+o(1) \\
\leq & \left(\int_{\left\{x \in \Lambda_{\varepsilon}:\left|u_{n}(x)\right| \geq r_{1}\right\}}\left(f\left(\varepsilon x,\left|u_{n}\right|\right)\left|u_{n}\right|\right)^{\sigma_{0}}\right)^{1 / \sigma_{0}} \cdot\left|u_{n}^{+}-u_{n}^{-}\right|_{p} \\
& +o(1) .
\end{aligned}
$$

It follows from $(2.7),(2.10)$ and $E$ embeds continuously into $L^{p}$, we find

$$
\frac{a-|V|_{\infty}}{4 a}\left\|u_{n}\right\|^{2} \leq C_{1}\left\|u_{n}\right\|+o(1) .
$$

Then $\left\{u_{n}\right\}$ is bounded in $E$ as desired.

Case 2. $\left(f_{4}\right)(i i)$ occurs.

Assume contrarily that $\left\|u_{n}\right\| \rightarrow \infty$ as $n \rightarrow \infty$ and set $v_{n}=u_{n} /\left\|u_{n}\right\|$. 
Then $\left|v_{n}\right|_{2}^{2} \leq C_{2}$ and $\left|v_{n}\right|_{3}^{2} \leq C_{3}$. It follows from (2.2) and (2.11) that

$$
\begin{aligned}
o(1)= & \left\|u_{n}\right\|^{2}\left(\left\|v_{n}\right\|^{2}+\Re \int V_{\varepsilon}(x) v_{n} \cdot \overline{\left(v_{n}^{+}-v_{n}^{-}\right)}\right. \\
& \left.-\Re \int f\left(\varepsilon x,\left|u_{n}\right|\right) v_{n} \cdot \overline{\left(v_{n}^{+}-v_{n}^{-}\right)}\right) \\
\geq & \left\|u_{n}\right\|^{2}\left(\frac{a-|V|_{\infty}}{a}-\Re \int f\left(\varepsilon x,\left|u_{n}\right|\right) v_{n} \cdot \overline{\left(v_{n}^{+}-v_{n}^{-}\right)}\right) .
\end{aligned}
$$

Thus

$$
\liminf _{n \rightarrow \infty} \Re \int f\left(\varepsilon x,\left|u_{n}\right|\right) v_{n} \cdot \overline{\left(v_{n}^{+}-v_{n}^{-}\right)} \geq \ell:=\frac{a-|V|_{\infty}}{a} .
$$

To get a contradiction, let us first set

$$
\begin{gathered}
d(r):=\inf \left\{\widehat{F}(\varepsilon x, s): x \in \mathbb{R}^{3}, \text { and } s>r\right\}, \\
\Omega_{n}(\rho, r):=\left\{x \in \mathbb{R}^{3}: \rho \leq\left|u_{n}(x)\right|<r\right\},
\end{gathered}
$$

and

$$
c_{\rho}^{r}:=\inf \left\{\frac{\widehat{F}(\varepsilon x, s)}{s^{2}}: x \in \mathbb{R}^{3}, \text { and } \rho \leq s \leq r\right\} .
$$

By $\left(f_{6}\right), d(r) \rightarrow \infty$ as $r \rightarrow \infty$ and by definition

$$
\widehat{F}\left(\varepsilon x, u_{n}(x)\right) \geq c_{\rho}^{r}\left|u_{n}(x)\right|^{2} \quad \text { for all } x \in \Omega_{n}(\rho, r) .
$$

From (2.10), we have

$$
C \geq \int_{\Omega_{n}(0, \rho)} \widehat{F}\left(\varepsilon x, u_{n}(x)\right)+c_{\rho}^{r} \int_{\Omega_{n}(\rho, r)}\left|u_{n}\right|^{2}+d(r)\left|\Omega_{n}(r, \infty)\right| .
$$

Observe that $\left|\Omega_{n}(r, \infty)\right| \leq C / d(r) \rightarrow 0$ as $r \rightarrow \infty$ unformly in $n$, and, for any fixed $0<\rho<r$,

$$
\int_{\Omega_{n}(\rho, r)}\left|v_{n}\right|^{2}=\frac{1}{\left\|u_{n}\right\|^{2}} \int_{\Omega_{n}(\rho, r)}\left|u_{n}\right|^{2} \leq \frac{C}{c_{\rho}^{r}\left\|u_{n}\right\|^{2}} \rightarrow 0
$$

as $n \rightarrow \infty$.

Now let us choose $0<\delta<\ell / 3$. By $\left(f_{1}\right)$ there is $\rho_{\delta}>0$ such that $|f(\varepsilon x, s)|<\frac{\delta}{C_{2}}$ for all $s \in\left[0, \rho_{\delta}\right]$. Consequently,

$$
\int_{\Omega_{n}(0, \rho)}\left|f\left(\varepsilon x,\left|u_{n}\right|\right)\right| \cdot\left|v_{n}\right| \cdot\left|v_{n}^{+}-v_{n}^{-}\right| \leq \frac{\delta}{C_{2}}\left|v_{n}\right|_{2}^{2} \leq \delta
$$


for all $n$. Recall that, by $\left(g_{1}\right),\left(g_{2}^{\prime}\right)$ and $(2.6), f(\varepsilon x, s) \leq b$ for all $(x, s)$. Using Hölder inequality we can take $r_{\delta}$ large so that

$$
\begin{aligned}
& \int_{\Omega_{n}\left(r_{\delta}, \infty\right)}\left|f\left(\varepsilon x,\left|u_{n}\right|\right)\right| \cdot\left|v_{n}\right| \cdot\left|v_{n}^{+}-v_{n}^{-}\right| \\
\leq & b \int_{\Omega_{n}\left(r_{\delta}, \infty\right)}\left|v_{n}\right| \cdot\left|v_{n}^{+}-v_{n}^{-}\right| \\
\leq & b \cdot\left|\Omega_{n}\left(r_{\delta}, \infty\right)\right|^{1 / 6} \cdot\left|v_{n}\right|_{2} \cdot\left|v_{n}^{+}-v_{n}^{-}\right|_{3} \\
\leq & C_{b}\left|\Omega_{n}\left(r_{\delta}, \infty\right)\right|^{1 / 6} \leq \delta
\end{aligned}
$$

for all $n$. Moreover, there is $n_{0}$ such that

$$
\begin{aligned}
& \int_{\Omega_{n}\left(\rho_{\delta}, r_{\delta}\right)}\left|f\left(\varepsilon x,\left|u_{n}\right|\right)\right| \cdot\left|v_{n}\right| \cdot\left|v_{n}^{+}-v_{n}^{-}\right| \\
\leq & b \int_{\Omega_{n}\left(\rho_{\delta}, r_{\delta}\right)}\left|v_{n}\right| \cdot\left|v_{n}^{+}-v_{n}^{-}\right| \\
\leq & b \cdot\left|v_{n}\right|_{2}\left(\int_{\Omega_{n}\left(\rho_{\delta}, r_{\delta}\right)}\left|v_{n}\right|^{2}\right)^{1 / 2} \leq \delta
\end{aligned}
$$

for all $n \geq n_{0}$. Therefore, for $n \geq n_{0}$,

$$
\int f\left(\varepsilon x,\left|u_{n}\right|\right)\left|v_{n}\right|\left|v_{n}^{+}-v_{n}^{-}\right| \leq 3 \delta<\ell,
$$

which contradicts (2.13).

To prove the compactness, we recall some direct observations: since $E$ embeds compactly into $L_{l o c}^{q}$ for all $q \in[1,3)$, the boundedness of $\left\{u_{n}\right\}$ implies that there exists $u \in E$ satisfying (after passing to a subsequence if necessary)

$$
u_{n} \rightarrow u \text { in } E, \quad u_{n} \rightarrow u \text { in } L_{l o c}^{q}
$$

for $q \in[1,3)$. Set $z_{n}=u_{n}-u$, we remark that $\left\{z_{n}\right\}$ is bounded and $z_{n} \rightarrow 0$ in $E$ and $z_{n} \rightarrow 0$ in $L_{l o c}^{q}$ as $n \rightarrow \infty$ for $q \in[1,3)$.

We conclude from $\left\{u_{n}\right\}$ is a bounded (P.S.) sequence that

$$
\begin{gathered}
o(1)=\left\langle u_{n}^{+}, z_{n}^{+}\right\rangle+\Re \int V_{\varepsilon}(x) u_{n} \cdot \overline{z_{n}^{+}}-\Re \int f\left(\varepsilon x,\left|u_{n}\right|\right) u_{n} \cdot \overline{z_{n}^{+}}, \\
0=\left\langle u^{+}, z_{n}^{+}\right\rangle+\Re \int V_{\varepsilon}(x) u \cdot \overline{z_{n}^{+}}-\Re \int f(\varepsilon x,|u|) u \cdot \overline{z_{n}^{+}}, \\
o(1)=-\left\langle u_{n}^{-}, z_{n}^{-}\right\rangle+\Re \int V_{\varepsilon}(x) u_{n} \cdot \overline{z_{n}^{-}}-\Re \int f\left(\varepsilon x,\left|u_{n}\right|\right) u_{n} \cdot \overline{z_{n}^{-}}, \\
0=-\left\langle u^{-}, z_{n}^{-}\right\rangle+\Re \int V_{\varepsilon}(x) u \cdot \overline{z_{n}^{-}}-\Re \int f(\varepsilon x,|u|) u \cdot \overline{z_{n}^{-}} .
\end{gathered}
$$


On the other hand, we find

$$
\left\{\begin{array}{l}
\Re \int f(\varepsilon x,|u|) u \cdot \overline{z_{n}^{+}}=o(1), \\
\Re \int f(\varepsilon x,|u|) u \cdot \overline{z_{n}^{-}}=o(1), \\
\Re \int f\left(\varepsilon x,\left|u_{n}\right|\right) u \cdot \overline{\left(z_{n}^{+}-z_{n}^{-}\right)}=o(1) .
\end{array}\right.
$$

Therefore, again with the definition of $f$, we deduce from (2.14)-(2.18) that

$$
\frac{a-|V|_{\infty}}{4 a}\left\|z_{n}\right\|^{2} \leq \Re \int_{\Lambda_{\varepsilon}} f\left(\varepsilon x,\left|u_{n}\right|\right) z_{n} \cdot \overline{\left(z_{n}^{+}-z_{n}^{-}\right)}+o(1) .
$$

Since $\Lambda_{\varepsilon}$ is bounded for any fixed $\varepsilon$, we have $\left\|z_{n}\right\|=o(1)$ as $n \rightarrow \infty$, which completes the proof.

The pervious lemma makes it possible to use the critical point theory to find critical points of $\widetilde{\Phi}_{\varepsilon}$. We will formulate an appropriate minimax level for $\widetilde{\Phi}_{\varepsilon}$.

First set, for $r>0, B_{r}=\{u \in E:\|u\| \leq r\}$, and for $e \in E^{+} \backslash\{0\}$

$$
E_{e}:=E^{-} \oplus \mathbb{R}^{+} e
$$

with $\mathbb{R}^{+}=[0,+\infty)$. It follows from $\left(g_{1}\right)$ and $\left(f_{2}\right)$ that there exists $C>0$ such that

$$
F(x, s) \leq \frac{a-|V|_{\infty}}{4} s^{2}+C s^{p} \quad \text { for all } s \geq 0 .
$$

So we have:

Lemma 2.5. There are $r>0$ and $\tau>0$, both independent of $\varepsilon$, such that $\left.\widetilde{\Phi}_{\varepsilon}\right|_{B_{r}^{+}} \geq 0$ and $\left.\widetilde{\Phi}_{\varepsilon}\right|_{S_{r}^{+}} \geq \tau$, where

$$
\begin{gathered}
B_{r}^{+}=B_{r} \cap E^{+}=\left\{u \in E^{+}:\|u\| \leq r\right\}, \\
S_{r}^{+}=\partial B_{r}^{+}=\left\{u \in E^{+}:\|u\|=r\right\} .
\end{gathered}
$$

Proof. Recall that $|u|_{p}^{p} \leq C_{p}\|u\|^{p}$ for all $u \in E$ by Sobolev's embedding theorem. The conclusion follows easily because, for $u \in E^{+}$

$$
\begin{aligned}
\widetilde{\Phi}_{\varepsilon}(u) & =\frac{1}{2}\|u\|^{2}-\frac{1}{2} \int V_{\varepsilon}(x)|u|^{2}-\Psi_{\varepsilon}(u) \\
& \geq \frac{1}{2}\|u\|^{2}-\frac{|V|_{\infty}}{2}|u|_{2}^{2}-\left(\frac{a-|V|_{\infty}}{4}|u|_{2}^{2}+C|u|_{p}^{p}\right) \\
& \geq \frac{a-|V|_{\infty}}{4 a}\|u\|^{2}-C^{\prime}\|u\|^{p}
\end{aligned}
$$

with $C, C^{\prime}>0$ independent of $u$ and $p>2$ (see (2.19)). 
Let $\mathscr{K}_{\varepsilon}:=\left\{u \in E \backslash\{0\}: \widetilde{\Phi}_{\varepsilon}^{\prime}(u)=0\right\}$ be the critical set of $\widetilde{\Phi}_{\varepsilon}$. By virtue of Lemma 2.4, using the same iterative argument of [18, Proposition 3.2] and [15, Lemma 3.19], we obtain the following

Lemma 2.6. If $u \in \mathscr{K}_{\varepsilon}$ with $\left|\widetilde{\Phi}_{\varepsilon}(u)\right| \leq C$. Then, for any $q \geq 2, u \in$ $W^{1, q}\left(\mathbb{R}^{3}, \mathbb{C}^{4}\right)$ with $\|u\|_{W^{1, q}} \leq C_{q}$, where $C_{q}$ depends only on $C$ and $q$;

Remark 2.7. Let $\mathscr{L}_{\varepsilon}$ be the set of all least energy solutions of $\widetilde{\Phi}_{\varepsilon}$. Let $u \in \mathscr{L}_{\varepsilon}$, then $\left|\widetilde{\Phi}_{\varepsilon}(u)\right| \leq C$ for some constant $C>0$ (this will be proved in Lemma 3.9). Therefore, as a consequence of Lemma 2.6, we see (together with the Sobolev embedding theorem) that there exist $C_{\infty}>0$ independent of $\varepsilon$ such that

$$
|u|_{\infty} \leq C_{\infty} \text { for all } u \in \mathscr{L}_{\varepsilon}
$$

\section{Some auxiliary results}

Firstly, it is easily checked that, for any $x_{0} \in \mathbb{R}^{3}$, setting $\tilde{V}_{\varepsilon}(x)=V(\varepsilon(x+$ $\left.x_{0}\right)$ ), if $\tilde{u}$ is a solution of

$$
-i \alpha \cdot \nabla \tilde{u}+a \beta \tilde{u}+\tilde{V}_{\varepsilon}(x) \tilde{u}=g(|\tilde{u}|) \tilde{u}
$$

then $u(x)=\tilde{u}\left(x-x_{0}\right)$ solves (1.8). Thus, without loss of generality, we can assume that $0 \in \Lambda$ and $V(0)=\min _{\Lambda} V$.

\subsection{Functional reduction}

Let us mention here that a reduction of an strongly indefinite functional to a functional on $E^{+}$is well know under stronger differentiability conditions, see for example $[1,26,27]$ and the papers concerned with Dirac operator. In $[26,27]$ a reduction in two steps has been performed: first to $E^{+}$and then to a Nehari manifold on $E^{+}$.

Motivated by the above papers, we shall use the reduction approaches to find critical points of $\Phi_{\varepsilon}$. Suppose $\left(f_{1}\right)-\left(f_{6}\right)$ are satisfied, for a fixed $u \in E^{+}$, let $\phi_{u}: E^{-} \rightarrow \mathbb{R}$ defined by $\phi_{u}(v)=\widetilde{\Phi}_{\varepsilon}(u+v)$. We infer

$$
\phi_{u}(v) \leq \frac{a+|V|_{\infty}}{2 a}\|u\|^{2}-\frac{a-|V|_{\infty}}{2 a}\|v\|^{2} .
$$

Moreover, we have, for any $v, w \in E^{-}$,

$$
\begin{aligned}
\phi_{u}^{\prime \prime}(v)[w, w] & =-\|w\|^{2}-\int V_{\varepsilon}(x)|w|^{2}-\Psi_{\varepsilon}^{\prime \prime}(u+v)[w, w] \\
& \leq-\frac{a-|V|_{\infty}}{a}\|w\|^{2} .
\end{aligned}
$$


Indeed, a direct calculation shows that

$$
\begin{aligned}
\Psi_{\varepsilon}^{\prime \prime}(u+v)[w, w]= & \int\left[f_{s}(\varepsilon x,|u+v|)|u+v|\left(\frac{(u+v) \cdot \bar{w}}{|u+v| \cdot|w|}\right)^{2}\right. \\
& +f(\varepsilon x,|u+v|)]|w|^{2} d x
\end{aligned}
$$

Then, it follows from $\left(f_{5}\right)$ and $\left(\frac{(u+v) \cdot \bar{w}}{|u+v| \cdot|w|}\right)^{2} \leq 1$ that $\Psi_{\varepsilon}^{\prime \prime}(u+v)[w, w] \geq 0$. As a consequence of (3.1) and (3.2) (that is anti-coercion and concavity), there exists a unique $h_{\varepsilon}: E^{+} \rightarrow E^{-}$such that

$$
\widetilde{\Phi}_{\varepsilon}\left(u+h_{\varepsilon}(u)\right)=\max _{v \in E^{-}} \widetilde{\Phi}_{\varepsilon}(u+v) .
$$

From the definition of $h_{\varepsilon}$, we have

$$
\begin{aligned}
0 \leq & \widetilde{\Phi}_{\varepsilon}\left(u+h_{\varepsilon}(u)\right)-\widetilde{\Phi}_{\varepsilon}(u) \\
= & -\frac{1}{2}\left\|h_{\varepsilon}(u)\right\|^{2}+\frac{1}{2} \int V_{\varepsilon}(x)\left|u+h_{\varepsilon}(u)\right|^{2}-\Psi_{\varepsilon}\left(u+h_{\varepsilon}(u)\right) \\
& -\frac{1}{2} \int V_{\varepsilon}(x)|u|^{2}+\Psi_{\varepsilon}(u) \\
\leq & -\frac{a-|V|_{\infty}}{2 a}\left\|h_{\varepsilon}(u)\right\|^{2}+\frac{|V|_{\infty}}{a}\|u\|^{2}+\Psi_{\varepsilon}(u)
\end{aligned}
$$

for all $u \in E^{+}$. Hence the boundedness of $\Psi_{\varepsilon}$ implies that of $h_{\varepsilon}$. Set $\pi: E^{+} \oplus E^{-} \rightarrow E^{-}$by

$$
\pi(u, v)=P^{-} \circ \mathcal{R} \circ \widetilde{\Phi}_{\varepsilon}^{\prime}(u+v),
$$

where $P^{-}: E \rightarrow E^{-}$is the projection and $\mathcal{R}: E^{*} \rightarrow E$ denotes the isomorphism induced from the Riesz representation theorem. Noting that for every $u \in E^{+}$, we have

$$
\pi\left(u, h_{\varepsilon}(u)\right)=0 .
$$

Since $\pi_{v}(u, v)=\left.P^{-} \circ \mathcal{R} \circ \widetilde{\Phi}_{\varepsilon}^{\prime \prime}(u+v)\right|_{E^{-}}$, from (3.2) it follows that $\pi_{v}\left(u, h_{\varepsilon}(u)\right)$ is an isomorphism with

$$
\left\|\pi_{v}\left(u, h_{\varepsilon}(u)\right)^{-1}\right\| \leq \frac{a}{a-|V|_{\infty}}
$$

for every $u \in E^{+}$. So (3.4) and (3.5) together with the implicit function theorem imply the uniquely defined map $h_{\varepsilon}: E^{+} \rightarrow E^{-}$is $C^{1}$ smooth with

$$
h_{\varepsilon}^{\prime}(u)=-\pi_{v}\left(u, h_{\varepsilon}(u)\right)^{-1} \circ \pi_{u}\left(u, h_{\varepsilon}(u)\right) \text {, }
$$

where $\pi_{u}(u, v):=\left.P^{-} \circ \mathcal{R} \circ \widetilde{\Phi}_{\varepsilon}^{\prime \prime}(u+v)\right|_{E^{+}}$. Define

$$
I_{\varepsilon}: E^{+} \rightarrow \mathbb{R}, \quad I_{\varepsilon}(u)=\widetilde{\Phi}_{\varepsilon}\left(u+h_{\varepsilon}(u)\right) .
$$


We see directly that critical points of $I_{\varepsilon}$ and $\widetilde{\Phi}_{\varepsilon}$ are in one-to-one correspondence via the injective map $u \mapsto u+h_{\varepsilon}(u)$ from $E^{+}$into $E$.

It is clear that for any $u \in E^{+}$and $v \in E^{-}$, by setting $z=v-h_{\varepsilon}(u)$ and $l(t)=\widetilde{\Phi}_{\varepsilon}\left(u+h_{\varepsilon}(u)+t z\right)$, one has $l(1)=\widetilde{\Phi}_{\varepsilon}(u+v), l(0)=\widetilde{\Phi}_{\varepsilon}\left(u+h_{\varepsilon}(u)\right)$ and $l^{\prime}(0)=0$. Hence we deduce $l(1)-l(0)=\int_{0}^{1}(1-s) l^{\prime \prime}(s) d s$. Consequently, we have

$$
\begin{aligned}
& \widetilde{\Phi}_{\varepsilon}(u+v)-\widetilde{\Phi}_{\varepsilon}\left(u+h_{\varepsilon}(u)\right) \\
= & \int_{0}^{1}(1-s) \widetilde{\Phi}_{\varepsilon}^{\prime \prime}\left(u+h_{\varepsilon}(u)+s z\right)[z, z] d s \\
= & -\int_{0}^{1}(1-s)\left(\|z\|^{2}+\int V_{\varepsilon}(x)|z|^{2} d x\right) d s \\
& -\int_{0}^{1}(1-s) \Psi_{\varepsilon}^{\prime \prime}\left(u+h_{\varepsilon}(u)+s z\right)[z, z] d s,
\end{aligned}
$$

which implies

$$
\begin{aligned}
& \int_{0}^{1}(1-s) \Psi_{\varepsilon}^{\prime \prime}\left(u+h_{\varepsilon}(u)+s z\right)[z, z] d s \\
& +\frac{1}{2}\|z\|^{2}+\frac{1}{2} \int V_{\varepsilon}(x)|z|^{2}=\widetilde{\Phi}_{\varepsilon}\left(u+h_{\varepsilon}(u)\right)-\widetilde{\Phi}_{\varepsilon}(u+v) .
\end{aligned}
$$

\subsection{The limit equation}

For $\mu \in(-a, a)$, assume $\left(g_{1}\right),\left(g_{3}\right)$ and either $\left(g_{2}\right)$ or $\left(g_{2}^{\prime}\right)$ are satisfied, let us consider the equation

$$
-i \alpha \cdot \nabla u+a \beta u+\mu u=g(|u|) u, \quad u \in H^{1}\left(\mathbb{R}^{3}, \mathbb{C}^{4}\right) .
$$

Its solutions are critical points of the functional

$$
\mathscr{T}_{\mu}(u):=\frac{1}{2}\left(\left\|u^{+}\right\|^{2}-\left\|u^{-}\right\|^{2}\right)+\frac{\mu}{2} \int|u|^{2}-\Psi(u)
$$

defined for $u=u^{+}+u^{-} \in E=E^{+} \oplus E^{-}$. Denote the critical set, the least energy and the set of least energy solutions of $\mathscr{T}_{\mu}$ as follows

$$
\begin{aligned}
& \mathscr{K}_{\mu}:=\left\{u \in E: \mathscr{T}_{\mu}^{\prime}(u)=0\right\} \\
& \gamma_{\mu}:=\inf \left\{\mathscr{T}_{\mu}(u): u \in \mathscr{K}_{\mu} \backslash\{0\}\right\}, \\
& \mathscr{R}_{\mu}:=\left\{u \in \mathscr{K}_{\mu}: \mathscr{T}_{\mu}(u)=\gamma_{\mu},|u(0)|=|u|_{\infty}\right\} .
\end{aligned}
$$

And as before, we introduce the following notations:

$$
\begin{aligned}
& \mathscr{J}_{\mu}: E^{+} \rightarrow E^{-}, \quad \mathscr{T}_{\mu}\left(u+\mathscr{J}_{\mu}(u)\right)=\max _{v \in E^{-}} \mathscr{T}_{\mu}(u+v) ; \\
& J_{\mu}: E^{+} \rightarrow \mathbb{R}, \quad J_{\mu}=\mathscr{T}_{\mu}\left(u+\mathscr{J}_{\mu}(u)\right) .
\end{aligned}
$$


Remark that, from the definition of $\mathscr{J}_{\mu}$, we have

$$
\mathscr{T}_{\mu}^{\prime}\left(u+\mathscr{J}_{\mu}(u)\right) z=0 \quad \text { for all } z \in E^{-} .
$$

And, similar to (3.3), there holds

$$
\left\|\mathscr{J}_{\mu}(u)\right\|^{2} \leq \frac{2|\mu|}{a-|\mu|}\|u\|^{2}+\frac{2 a}{a-|\mu|} \Psi_{\varepsilon}(u) .
$$

\subsubsection{The super-linear case}

The following lemma is from [9] (see also [14])

Lemma 3.1. For the equation (3.8) we have:

(i) $\mathscr{K}_{\mu} \backslash\{0\} \neq \emptyset, \gamma_{\mu}>0$ and $\mathscr{K}_{\mu} \subset \cap_{q \geq 2} W^{1, q}$;

(ii) $\gamma_{\mu}$ is attained and $\mathscr{R}_{\mu}$ is compact in $H^{1}\left(\mathbb{R}^{3}, \mathbb{C}^{4}\right)$;

(iii) there exist $C, c>0$ such that

$$
|w(x)| \leq C \exp (-c|x|)
$$

for all $x \in \mathbb{R}^{3}$ and $w \in \mathscr{R}_{\mu}$.

Observe that assumption $\left(g_{2}\right)(i i)$ implies that for any $\delta>0$ there is a constant $c_{\delta}>0$ such that

$$
G(s) \geq c_{\delta} s^{\theta}-\delta s^{2} \quad \text { for all } s \geq 0 .
$$

Directly, for $v \in E^{-}, u=t e+v \in E_{e}$, we have that

$$
\begin{aligned}
\mathscr{T}_{\mu}(u) & =\frac{t^{2}}{2}\|e\|^{2}-\frac{\|v\|^{2}}{2}+\frac{\mu}{2} \int|t e+v|^{2}-\int G(|t e+v|) \\
& \leq \frac{a+|\mu+2 \delta|}{2 a} t^{2}\|e\|^{2}-\frac{a-|\mu+2 \delta|}{2 a}\|v\|^{2}-c_{\delta} \int|t e+v|^{\theta} .
\end{aligned}
$$

And hence, by Proposition 2.1,

$$
\mathscr{T}_{\mu}(u) \leq \frac{a+|\mu+2 \delta|}{2 a} t^{2}\|e\|^{2}-\frac{a-|\mu+2 \delta|}{2 a}\|v\|^{2}-C_{\delta, \theta} t^{\theta}|e|_{\theta}^{\theta} \quad \text { on } E_{e} .
$$

As a consequence of the above estimates we have the following lemma, the specific proofs can be found in [12] (see also [10, 14])

Lemma 3.2. There hold the following properties:

(1) For any $e \in E^{+} \backslash\{0\}$, we have $\mathscr{T}_{\mu}(u) \rightarrow-\infty$ provided $u \in E_{e}$ and $\|u\| \rightarrow \infty$ 
(2) Set $\Gamma_{\mu}=\left\{\nu \in C\left([0,1], E^{+}\right): \nu(0)=0, J_{\mu}(\nu(1))<0\right\}$, we have

$$
\gamma_{\mu}=\inf _{\nu \in \Gamma_{\mu}} \max _{t \in[0,1]} J_{\mu}(\nu(t))=\inf _{u \in E^{+} \backslash\{0\}} \max _{t \geq 0} J_{\mu}(t u) .
$$

(3) If $\mu_{1}>\mu_{2}$, then $\gamma_{\mu_{1}}>\gamma_{\mu_{2}}$.

Notice that, similar to (3.7), we have for $u \in E^{+}, v \in E^{-}$and $z=$ $v-\mathscr{J}_{\mu}(u)$

$$
\begin{aligned}
& \int_{0}^{1}(1-s) \Psi^{\prime \prime}\left(u+\mathscr{J}_{\mu}(u)+s z\right)[z, z] d s \\
& +\frac{1}{2}\|z\|^{2}+\frac{\mu}{2} \int|z|^{2}=\mathscr{T}_{\mu}\left(u+\mathscr{J}_{\mu}(u)\right)-\mathscr{T}_{\mu}(u+v) .
\end{aligned}
$$

\subsubsection{The asymptotically linear case}

Firstly, let $\left(E_{s}\right)_{s \in \mathbb{R}}$ denote the spectral family of $H_{0}:=-i \alpha \cdot \nabla+a \beta$. Choose a number $a+|V|_{\infty}<\kappa<b$. Since $H_{0}$ is invariant under the action of $\mathbb{Z}^{3}$, the subspace $Y_{0}:=\left(E_{\kappa}-E_{0}\right) L^{2}$ is infinite-dimensional, and

$$
a|u|_{2}^{2} \leq\|u\|^{2} \leq \kappa|u|_{2}^{2} \quad \text { for all } u \in Y_{0} .
$$

Take an element $e \in Y_{0}$ arbitrarily, we have

Lemma 3.3. There holds the following:

(1) $\sup \mathscr{T}_{\mu}\left(E_{e}\right)<+\infty$, and $\mathscr{T}_{\mu}(u) \rightarrow-\infty$ provided $u \in E_{e}$ and $\|u\| \rightarrow \infty$.

(2) For any $u \in E^{+} \backslash\{0\}$, taking $t \rightarrow \infty$, then either $J_{\mu}(t u) \rightarrow+\infty$ or $J_{\mu}(t u) \rightarrow-\infty$.

Proof. For the proof of (1), we refer [9, Lemma 7.7]. To show (2), let us first assume $\sup _{t \geq 0} J_{\mu}(t u)=M<+\infty$. Following (3.9), a direct calculation shows

$$
\begin{aligned}
\frac{d}{d t} J_{\mu}(t u) & =\frac{1}{t} J_{\mu}^{\prime}(t u) t u=\frac{1}{t} \mathscr{T}_{\mu}^{\prime}\left(t u+\mathscr{J}_{\mu}(t u)\right)\left(t u+\mathscr{J}_{\mu}^{\prime}(t u) t u\right) \\
& =\frac{1}{t} \mathscr{T}_{\mu}^{\prime}\left(t u+\mathscr{J}_{\mu}(t u)\right)\left(t u+\mathscr{J}_{\mu}(t u)\right) \\
& =\frac{2 J_{\mu}(t u)}{t}-\frac{2}{t} \int \widehat{G}\left(\left|t u+\mathscr{J}_{\mu}(t u)\right|\right) .
\end{aligned}
$$

For $r>0$, we infer

$$
\begin{aligned}
& \int \widehat{G}\left(\left|t u+\mathscr{J}_{\mu}(t u)\right|\right) \\
\geq & \int_{\left\{x \in \mathbb{R}^{3}:\left|u+\mathscr{J}_{\mu}(t u) / t\right| \geq r\right\}} \widehat{G}\left(\left|t u+\mathscr{J}_{\mu}(t u)\right|\right) \\
\geq & \widehat{G}(r t) \cdot \operatorname{meas}\left\{x \in \mathbb{R}^{3}:\left|u+\mathscr{J}_{\mu}(t u) / t\right| \geq r\right\} .
\end{aligned}
$$


Since the family $\left\{\mathscr{J}_{\mu}(t u) / t\right\}_{t>0} \subset E^{-}$is bounded (due to (3.10) and $\left(g_{2}^{\prime}\right)(i)$ ), we must have meas $\left\{x \in \mathbb{R}^{3}:\left|u+\mathscr{J}_{\mu}(t u) / t\right| \geq r\right\} \geq \bar{\delta}$ with some $\bar{\delta}>0$ for all $t>0$ provided $r>0$ is small. Indeed, if such $\bar{\delta}$ does not exist, we then have

$$
\mathscr{J}_{\mu}\left(t_{j} u\right) / t_{j} \rightarrow-u \text { in } E
$$

for some sequence $\left\{t_{j}\right\}$. However, this will imply $u=0$ since $u \in E^{+}$, which is a contradiction. Now, from $(3.14)$ and $\left(g_{2}^{\prime}\right)(i i)$, we deduce that

$$
\begin{aligned}
\frac{d}{d t} J_{\mu}(t u) & \leq \frac{2 J_{\mu}(t u)}{t}-2 \bar{\delta} \cdot \frac{\widehat{G}(r t)}{t} \\
& \leq \frac{2 J_{\mu}(t u)}{t}-\frac{3 M}{t} \\
& \leq-\frac{M}{t}
\end{aligned}
$$

for $t$ sufficiently large. Thus we have $J_{\mu}(t u)=\int_{0}^{t} \frac{d}{d t} J_{\mu}(t u) \rightarrow-\infty$ as $t \rightarrow$ $+\infty$.

As in Lemma 3.2, let us consider the family

$$
\Gamma_{\mu}=\left\{\nu \in C\left([0,1], E^{+}\right): \nu(0)=0, J_{\mu}(\nu(1))<0\right\},
$$

and the minimax schemes

$$
d_{\mu}^{1}=\inf _{\nu \in \Gamma_{\mu}} \max _{t \in[0,1]} J_{\mu}(\nu(t)) \quad \text { and } \quad d_{\mu}^{2}=\inf _{u \in E+\backslash\{0\}} \max _{t \geq 0} J_{\mu}(t u) .
$$

Lemma 3.4. For the asymptotically linear equation (3.8), there holds:

(1) $\mathscr{K}_{\mu} \backslash\{0\} \neq \emptyset, \gamma_{\mu}>0$ and $\mathscr{K}_{\mu} \subset \cap_{q \geq 2} W^{1, q}$;

(2) $\gamma_{\mu}$ is attained and $\gamma_{\mu}=d_{\mu}^{1}=d_{\mu}^{2}$;

(3) if $\mu_{1}>\mu_{2}$, then $\gamma_{\mu_{1}}>\gamma_{\mu_{2}}$.

Proof. Since (1) is a direct consequence of [9, Theorem 7.3], we only need to prove (2) and (3).

To show (2), assume $\left\{u_{n}\right\} \subset \mathscr{K}_{\mu} \backslash\{0\}$ such that $\mathscr{T}_{\mu}\left(u_{n}\right) \rightarrow \gamma_{\mu}$. Clearly $\left\{u_{n}\right\}$ is a $(C)_{c}$ sequence, hence is bounded. As is proved in [9], $\left\{u_{n}\right\}$ is non-vanishing. Since $\mathscr{T}_{\mu}$ is $\mathbb{Z}^{3}$-invariant, up to a translation, we can assume $u_{n} \rightarrow u \in \mathscr{K}_{\mu} \backslash\{0\}$. Observe that, by Fatou's lemma,

$$
\begin{aligned}
\gamma_{\mu} & \leq \mathscr{T}_{\mu}(u)=\mathscr{T}_{\mu}(u)-\frac{1}{2} \mathscr{T}_{\mu}^{\prime}(u) u=\int \widehat{G}(|u|) \\
& \leq \liminf _{n \rightarrow \infty} \int \widehat{G}\left(\left|u_{n}\right|\right)=\liminf _{n \rightarrow \infty}\left(\mathscr{T}_{\mu}\left(u_{n}\right)-\frac{1}{2} \mathscr{T}_{\mu}^{\prime}\left(u_{n}\right) u_{n}\right) \\
& =\gamma_{\mu},
\end{aligned}
$$


we find $\gamma_{\mu}$ is attained. By noting that $\gamma_{\mu}$ is also the least energy of $J_{\mu}$, it is standard to check that $\gamma_{\mu} \leq d_{\mu}^{1} \leq d_{\mu}^{2}$. To prove $d_{\mu}^{2} \leq \gamma_{\mu}$ we first note that if $s>0$, by virtue of $\left(g_{1}\right), g^{\prime}(s) s>0$. Hence, if $u \in E \backslash\{0\}$ and $v \in E$, we have

$$
\begin{aligned}
& \left(\Psi^{\prime \prime}(u)[u, u]-\Psi^{\prime}(u) u\right)+2\left(\Psi^{\prime \prime}(u)[u, v]-\Psi^{\prime}(u) v\right)+\Psi^{\prime \prime}(u)[v, v] \\
= & \int g(|u|)|v|^{2}+\int g^{\prime}(|u|)|u|\left(|u|+\frac{\Re u \cdot v}{|u|}\right)^{2}>0 .
\end{aligned}
$$

As a consequence of [1, Theorem 5.1], if $z \in E^{+} \backslash\{0\}$ satisfies $J_{\mu}^{\prime}(z) z=0$ then $J_{\mu}^{\prime \prime}(z)[z, z]<0$. Therefore, let $u \in E^{+} \backslash\{0\}$, we find the function $t \mapsto J_{\mu}(t u)$ has at most one nontrivial critical point $t=t(u)>0$. So, denoted by

$$
\mathscr{M}_{\mu}:=\left\{t(u) u: u \in E^{+} \backslash\{0\}, t(u)<\infty\right\},
$$

we have $\mathscr{M}_{\mu} \neq \emptyset$ due to $\gamma_{\mu}$ is attained. Meanwhile, we notice

$$
d_{\mu}^{2}=\inf _{z \in \mathscr{M}_{\mu}} J_{\mu}(z) .
$$

Hence we have $d_{\mu}^{2} \leq \gamma_{\mu}$ since $u^{+} \in \mathscr{M}_{\mu}$ provided $u \in \mathscr{R}_{\mu}$.

Finally, (3) comes directly because, if $u \in \mathscr{R}_{\mu_{1}}$, we already have $u^{+}$is a critical point of $J_{\mu_{1}}$ and $\gamma_{\mu_{1}}=J_{\mu_{1}}\left(u^{+}\right)=\max _{t \geq 0} J_{\mu_{1}}\left(t u^{+}\right)$. Let $\tau>0$ such that $J_{\mu_{2}}\left(\tau u^{+}\right)=\max _{t \geq 0} J_{\mu_{2}}\left(t u^{+}\right)$, we deduce

$$
\begin{aligned}
\gamma_{\mu_{1}} & =J_{\mu_{1}}\left(u^{+}\right)=\max _{t \geq 0} J_{\mu_{1}}\left(t u^{+}\right) \\
& \geq J_{\mu_{1}}\left(\tau u^{+}\right)=\mathscr{T}_{\mu_{1}}\left(\tau u^{+}+\mathscr{J}_{\mu_{1}}\left(\tau u^{+}\right)\right) \\
& \geq \mathscr{T}_{\mu_{1}}\left(\tau u^{+}+\mathscr{J}_{\mu_{2}}\left(\tau u^{+}\right)\right) \\
& \geq \mathscr{T}_{\mu_{2}}\left(\tau u^{+}+\mathscr{J}_{\mu_{2}}\left(\tau u^{+}\right)\right)+\frac{\mu_{1}-\mu_{2}}{2}\left|\tau u^{+}+\mathscr{J}_{\mu_{2}}\left(\tau u^{+}\right)\right|_{2}^{2} \\
& =J_{\mu_{2}}\left(\tau u^{+}\right)+\frac{\mu_{1}-\mu_{2}}{2}\left|\tau u^{+}+\mathscr{J}_{\mu_{2}}\left(\tau u^{+}\right)\right|_{2}^{2} \\
& \geq \gamma_{\mu_{2}}+\frac{\mu_{1}-\mu_{2}}{2}\left|\tau u^{+}+\mathscr{J}_{\mu_{2}}\left(\tau u^{+}\right)\right|_{2}^{2},
\end{aligned}
$$

which ends the proof.

\subsection{Some technical results}

We remark that, by $\left(V_{1}\right), V_{\varepsilon}(x) \rightarrow V(0)$ uniformly on bounded sets of $\mathbb{R}^{3}$ as $\varepsilon \rightarrow 0$. We will make use of this property and the results just proved in Subsection 3.2 to prove some technical results that seem to be useful in the sequel.

Denote $V_{0}=V(0)$, set $V^{0}(x)=V(x)-V(0)$ and $V_{\varepsilon}^{0}(x)=V^{0}(\varepsilon x)$, we soon have

$$
\widetilde{\Phi}_{\varepsilon}(u)=\mathscr{T}_{V_{0}}(u)+\frac{1}{2} \int V_{\varepsilon}^{0}(x)|u|^{2}-\int(F(\varepsilon x,|u|)-G(|u|)) .
$$


Lemma 3.5. Let $\left(f_{1}\right)-\left(f_{5}\right)$ be satisfied, for each $u \in E^{+}$, we have $h_{\varepsilon}(u) \rightarrow$ $\mathscr{J}_{V_{0}}(u)$ as $\varepsilon \rightarrow 0$.

Proof. By (3.15), we deduce that

$$
\begin{aligned}
& \left(\widetilde{\Phi}_{\varepsilon}\left(z_{\varepsilon}\right)-\widetilde{\Phi}_{\varepsilon}(w)\right)+\left(\mathscr{T}_{V_{0}}(w)-\mathscr{T}_{V_{0}}\left(z_{\varepsilon}\right)\right) \\
= & \frac{1}{2} \int V_{\varepsilon}^{0}(x)\left(\left|z_{\varepsilon}\right|^{2}-|w|^{2}\right)+\int\left(G\left(\left|z_{\varepsilon}\right|\right)-G(|w|)\right) \\
& -\int\left(F\left(\varepsilon x,\left|z_{\varepsilon}\right|\right)-F(\varepsilon x,|w|)\right)
\end{aligned}
$$

where $z_{\varepsilon}=u+h_{\varepsilon}(u), w=u+\mathscr{J}_{V_{0}}(u)$. Denoted by $v_{\varepsilon}=z_{\varepsilon}-w$, we find

$$
\int V_{\varepsilon}^{0}(x)\left(\left|z_{\varepsilon}\right|^{2}-|w|^{2}\right)=\int V_{\varepsilon}^{0}(x)\left|v_{\varepsilon}\right|^{2}+2 \Re \int V_{\varepsilon}^{0}(x) w \cdot \overline{v_{\varepsilon}}
$$

and

$$
\begin{aligned}
& \int\left(G\left(\left|z_{\varepsilon}\right|\right)-G(|w|)\right)-\int\left(F\left(\varepsilon x,\left|z_{\varepsilon}\right|\right)-F(\varepsilon x,|w|)\right) \\
= & \Re \int g(|w|) w \cdot \overline{v_{\varepsilon}}-\Re \int f(\varepsilon x,|w|) w \cdot \overline{v_{\varepsilon}} \\
& +\int_{0}^{1}(1-s) \Psi^{\prime \prime}\left(w+s v_{\varepsilon}\right)\left[v_{\varepsilon}, v_{\varepsilon}\right] d s \\
& -\int_{0}^{1}(1-s) \Psi_{\varepsilon}^{\prime \prime}\left(w+s v_{\varepsilon}\right)\left[v_{\varepsilon}, v_{\varepsilon}\right] d s .
\end{aligned}
$$

Remark that, similar to (3.7) and (3.11), we infer

$$
\begin{aligned}
& \int_{0}^{1}(1-s) \Psi_{\varepsilon}^{\prime \prime}\left(z_{\varepsilon}-s v_{\varepsilon}\right)\left[v_{\varepsilon}, v_{\varepsilon}\right] d s \\
& +\frac{1}{2}\left\|v_{\varepsilon}\right\|^{2}+\frac{1}{2} \int V_{\varepsilon}(x)\left|v_{\varepsilon}\right|^{2}=\widetilde{\Phi}_{\varepsilon}\left(z_{\varepsilon}\right)-\widetilde{\Phi}_{\varepsilon}(w)
\end{aligned}
$$

and

$$
\begin{aligned}
& \int_{0}^{1}(1-s) \Psi^{\prime \prime}\left(w+s v_{\varepsilon}\right)\left[v_{\varepsilon}, v_{\varepsilon}\right] d s \\
& +\frac{1}{2}\left\|v_{\varepsilon}\right\|^{2}+\frac{V_{0}}{2}\left|v_{\varepsilon}\right|_{2}^{2}=\mathscr{T}_{V_{0}}(w)-\mathscr{T}_{V_{0}}\left(z_{\varepsilon}\right) .
\end{aligned}
$$


Then we get from (3.16) (jointly with the definition of $f$ and (2.19))

$$
\begin{aligned}
\left\|v_{\varepsilon}\right\|^{2}+V_{0}\left|v_{\varepsilon}\right|_{2}^{2} \leq & \Re \int V_{\varepsilon}^{0}(x) w \cdot \overline{v_{\varepsilon}}+\Re \int g(|w|) w \cdot \overline{v_{\varepsilon}} \\
& -\Re \int f(\varepsilon x,|w|) w \cdot \overline{v_{\varepsilon}} \\
\leq & \int\left|V_{\varepsilon}^{0}(x)\right| \cdot|w| \cdot\left|v_{\varepsilon}\right|+c_{1} \int_{\mathbb{R}^{3} \backslash \Lambda_{\varepsilon}}|w| \cdot\left|v_{\varepsilon}\right| \\
& +c_{1} \int_{\mathbb{R}^{3} \backslash \Lambda_{\varepsilon}}|w|^{p-1} \cdot\left|v_{\varepsilon}\right| \\
& +\frac{a-|V|_{\infty}}{2} \int_{\mathbb{R}^{3} \backslash \Lambda_{\varepsilon}}|w| \cdot\left|v_{\varepsilon}\right| \\
\leq & \left(\int\left|V_{\varepsilon}^{0}(x)\right|^{2}|w|^{2}\right)^{1 / 2}\left|v_{\varepsilon}\right|_{2} \\
& +c_{2}\left(\int_{\mathbb{R}^{3} \backslash \Lambda_{\varepsilon}}|w|^{2}\right)^{1 / 2}\left|v_{\varepsilon}\right|_{2} \\
& +c_{1}\left(\int_{\mathbb{R}^{3} \backslash \Lambda_{\varepsilon}}|w|^{p}\right)^{(p-1) / p}\left|v_{\varepsilon}\right|_{p} .
\end{aligned}
$$

Since $V_{\varepsilon}^{0}(x) \rightarrow 0$ uniformly on bounded sets of $\mathbb{R}^{3}$ as $\varepsilon \rightarrow 0$, we easily have

$$
\int\left|V_{\varepsilon}^{0}(x)\right|^{2}|w|^{2}=o(1)
$$

Moreover, by noting that $w$ decays at infinity in the sense for $q=2, p$,

$$
\limsup _{R \rightarrow \infty} \int_{|x| \geq R}|w|^{q}=0
$$

We find (due to $0 \in \Lambda$ )

$$
\begin{gathered}
\int_{\mathbb{R}^{3} \backslash \Lambda_{\varepsilon}}|w|^{2}=o(1), \\
\int_{\mathbb{R}^{3} \backslash \Lambda_{\varepsilon}}|w|^{p}=o(1),
\end{gathered}
$$

as $\varepsilon \rightarrow 0$. Thus (3.17) leads to $\left\|v_{\varepsilon}\right\|=\left\|h_{\varepsilon}(u)-\mathscr{J}_{V_{0}}(u)\right\|=o(1)$ as $\varepsilon \rightarrow 0$. So we have the lemma proved.

Lemma 3.6. Assume that $\left(f_{1}\right)-\left(f_{6}\right)$ are satisfied, for $\varepsilon>0$ small enough, $I_{\varepsilon}$ possesses the mountain-pass structure:

(1) $I_{\varepsilon}(0)=0$ and there exist $r>0$ and $\tau>0$ (both independent of $\varepsilon$ ) such that $\left.I_{\varepsilon}\right|_{S_{r}^{+}} \geq \tau$.

(2) there exists $u_{0} \in E^{+}$(independent of $\varepsilon$ ) such that $\left\|u_{0}\right\|>r$ and $I_{\varepsilon}\left(u_{0}\right)<0$. 
Proof. Since we have $I_{\varepsilon}(u) \geq \widetilde{\Phi}_{\varepsilon}(u)$ for all $u \in E^{+}$, (1) follows easily from Lemma 2.5.

To check (2), let $w=w^{+}+w^{-} \in \mathscr{R}_{V_{0}}$ be the least energy solution to

$$
-i \alpha \cdot \nabla u+a \beta u+V_{0} u=g(|u|) u
$$

with $|w(0)|=\max _{\mathbb{R}^{3}}|w(x)|$. Following Lemma 3.2 (2) and Lemma $3.4(2)$, we have

$$
\gamma_{V_{0}}=\inf _{\gamma \in \Gamma_{0}} \max _{t \in[0,1]} J_{V_{0}}(\gamma(t))=\inf _{e \in E^{+} \backslash\{0\}} \max _{t \geq 0} J_{V_{0}}(t u),
$$

where $\Gamma_{0}:=\left\{\gamma \in C\left([0,1], E^{+}\right): \gamma(0)=0, J_{V_{0}}(\gamma(1))<0\right\}$. From Lemma 3.2 (1) and Lemma $3.3(2)$, we see that there exists $t_{0}>0$ (large enough) such that

$$
\begin{aligned}
J_{V_{0}}\left(t_{0} w^{+}\right)= & \frac{1}{2}\left(\left\|t_{0} w^{+}\right\|^{2}-\left\|\mathscr{J}_{V_{0}}\left(t_{0} w^{+}\right)\right\|^{2}\right)+\frac{V_{0}}{2} \int\left|t_{0} w^{+}+\mathscr{J}_{V_{0}}\left(t_{0} w^{+}\right)\right|^{2} \\
& -\int G\left(\left|t_{0} w^{+}+\mathscr{J}_{V_{0}}\left(t_{0} w^{+}\right)\right|\right)<-1 .
\end{aligned}
$$

Hence, there is $R_{0}>0$ such that

$$
\begin{aligned}
& \frac{1}{2}\left(\left\|t_{0} w^{+}\right\|^{2}-\left\|\mathscr{J}_{V_{0}}\left(t_{0} w^{+}\right)\right\|^{2}\right)+\frac{V_{0}}{2} \int\left|t_{0} w^{+}+\mathscr{J}_{V_{0}}\left(t_{0} w^{+}\right)\right|^{2} \\
& -\int_{B_{R_{0}}} G\left(\left|t_{0} w^{+}+\mathscr{J}_{V_{0}}\left(t_{0} w^{+}\right)\right|\right) \leq-\frac{1}{2} .
\end{aligned}
$$

Recall that $V_{\varepsilon}(x) \rightarrow V_{0}$ uniformly on bounded sets of $\mathbb{R}^{3}$, it follows from Lemma 3.5 and (3.18) that

$$
\begin{aligned}
I_{\varepsilon}\left(t_{0} w^{+}\right)= & \frac{1}{2}\left(\left\|t_{0} w^{+}\right\|^{2}-\left\|h_{\varepsilon}\left(t_{0} w^{+}\right)\right\|^{2}\right)+\frac{1}{2} \int V_{\varepsilon}(x)\left|t_{0} w^{+}+h_{\varepsilon}\left(t_{0} w^{+}\right)\right|^{2} \\
& -\int F\left(\varepsilon x,\left|t_{0} w^{+}+h_{\varepsilon}\left(t_{0} w^{+}\right)\right|\right) \\
\leq & \frac{1}{2}\left(\left\|t_{0} w^{+}\right\|^{2}-\left\|h_{\varepsilon}\left(t_{0} w^{+}\right)\right\|^{2}\right)+\frac{1}{2} \int V_{\varepsilon}(x)\left|t_{0} w^{+}+h_{\varepsilon}\left(t_{0} w^{+}\right)\right|^{2} \\
& -\int_{\Lambda_{\varepsilon}} G\left(\left|t_{0} w^{+}+h_{\varepsilon}\left(t_{0} w^{+}\right)\right|\right) \\
\leq & \frac{1}{2}\left(\left\|t_{0} w^{+}\right\|^{2}-\left\|\mathscr{J}_{V_{0}}\left(t_{0} w^{+}\right)\right\|^{2}\right)+\frac{V_{0}}{2} \int\left|t_{0} w^{+}+\mathscr{J}_{V_{0}}\left(t_{0} w^{+}\right)\right|^{2} \\
& -\int_{B_{R_{0}}} G\left(\left|t_{0} w^{+}+\mathscr{J}_{V_{0}}\left(t_{0} w^{+}\right)\right|\right)+o(1) \\
\leq & -\frac{1}{2}+o(1) \quad \text { as } \varepsilon \rightarrow 0 .
\end{aligned}
$$

Therefore, there is $\varepsilon_{0}>0$ such that $I_{\varepsilon}\left(t_{0} w^{+}\right)<0$ for all $\varepsilon \in\left(0, \varepsilon_{0}\right]$, ends the proof. 
Lemma 3.7. Assuming $\left(f_{1}\right)-\left(f_{6}\right)$, for each $\varepsilon>0, I_{\varepsilon}$ satisfies the $(C)_{c^{-}}$ condition.

Proof. Firstly, it follows from the definition of $h_{\varepsilon}$ that

$$
\widetilde{\Phi}_{\varepsilon}^{\prime}\left(u+h_{\varepsilon}(u)\right) z=0 \quad \text { for all } u \in E^{+} \text {and } z \in E^{-} .
$$

Hence a direct calculation shows

$$
\begin{aligned}
I_{\varepsilon}^{\prime}(u) u & =\widetilde{\Phi}_{\varepsilon}^{\prime}\left(u+h_{\varepsilon}(u)\right)\left(u+h_{\varepsilon}^{\prime}(u) u\right) \\
& =\widetilde{\Phi}_{\varepsilon}^{\prime}\left(u+h_{\varepsilon}(u)\right)\left(u+h_{\varepsilon}(u)\right) \\
& =\widetilde{\Phi}_{\varepsilon}^{\prime}\left(u+h_{\varepsilon}(u)\right)\left(u-h_{\varepsilon}(u)\right) .
\end{aligned}
$$

Now let $\left\{w_{n}\right\} \subset E^{+}$be a $(C)_{c}$ sequence for $I_{\varepsilon}$ and set $u_{n}:=w_{n}+$ $h_{\varepsilon}\left(w_{n}\right)$, we check easily (from the proof of Lemma 2.4) that $\left\{u_{n}\right\}$ possesses a convergent subsequence. Therefore, we have $I_{\varepsilon}$ satisfies the $(C)_{c}$ condition.

Define

$$
c_{\varepsilon}:=\inf _{\nu \in \Gamma_{\varepsilon}} \max _{t \in[0,1]} I_{\varepsilon}(\nu(t)),
$$

where $\Gamma_{\varepsilon}:=\left\{\nu \in C\left([0,1], E^{+}\right): \nu(0)=0, I_{\varepsilon}(\nu(1))<0\right\}$. Then $\tau \leq c_{\varepsilon}<\infty$ is a well-defined critical value for $I_{\varepsilon}$ (also for $\widetilde{\Phi}_{\varepsilon}$ ).

Lemma 3.8. $c_{\varepsilon}=\inf _{u \in E^{+} \backslash\{0\}} \max _{t \geq 0} I_{\varepsilon}(t u)$.

Proof. Indeed, set $d_{\varepsilon}=\inf _{u \in E^{+} \backslash\{0\}} \max _{t \geq 0} I_{\varepsilon}(t u)$, we have $d_{\varepsilon} \geq c_{\varepsilon}$ by virtue of $\left(f_{6}\right)$ and the proof of Lemma $3.3(2)$. To prove the other inequality we first note that if $s>0$, by virtue of $\left(g_{1}\right),\left(g_{3}\right)$ and the definition of $f, f_{s}(x, s) s>0$. Hence, if $u \in E \backslash\{0\}$ and $v \in E$, we have

$$
\begin{aligned}
& \left(\Psi_{\varepsilon}^{\prime \prime}(u)[u, u]-\Psi_{\varepsilon}^{\prime}(u) u\right)+2\left(\Psi_{\varepsilon}^{\prime \prime}(u)[u, v]-\Psi_{\varepsilon}^{\prime}(u) v\right)+\Psi_{\varepsilon}^{\prime \prime}(u)[v, v] \\
= & \int f(\varepsilon x,|u|)|v|^{2}+\int f_{s}(\varepsilon x,|u|)|u|\left(|u|+\frac{\Re u \cdot v}{|u|}\right)^{2}>0 .
\end{aligned}
$$

As proved in [1], if $z \in E^{+} \backslash\{0\}$ satisfies $I_{\varepsilon}^{\prime}(z) z=0$ then $I_{\varepsilon}^{\prime \prime}(z)[z, z]<0$. Therefore, let $u \in E^{+} \backslash\{0\}$, we find the function $t \mapsto I_{\varepsilon}(t u)$ has at most one nontrivial critical point $t=t(u)>0$. So, denoted by

$$
\mathscr{N}:=\left\{t(u) u: u \in E^{+} \backslash\{0\}, t(u)<\infty\right\},
$$

we have $\mathscr{N} \neq \emptyset$ due to Lemma 3.6 and Lemma 3.7 (In general, we remark that $\mathscr{N}$ is not the Nehari manifold since $\mathscr{N}$ is not defined for all directions in $E^{+}$for the asymptotically linear case). Meanwhile, we notice

$$
d_{\varepsilon}=\inf _{z \in \mathscr{N}} I_{\varepsilon}(z) .
$$


Thus we only need to show that given $\nu \in \Gamma_{\varepsilon}$ there exists $\bar{t} \in[0,1]$ such that $\nu(\bar{t}) \in \mathscr{N}$. Assuming contrarily we have $\nu([0,1]) \cap \mathscr{N}=\emptyset$. In virtue of $\left(f_{1}\right)$ and Lemma 2.5

$$
I_{\varepsilon}^{\prime}(\nu(t)) \nu(t)>0 \text { for } t>0 \text { small . }
$$

Since the function $t \mapsto I_{\varepsilon}^{\prime}(\nu(t)) \nu(t)$ is continuous and $I_{\varepsilon}^{\prime}(\nu(t)) \nu(t) \neq 0$ for all $t \in(0,1]$, we have

$$
I_{\varepsilon}^{\prime}(\nu(t)) \nu(t)>0 \quad \text { for all } t \in(0,1] .
$$

Then, we find for all $t \in[0,1]$

$$
\begin{aligned}
I_{\varepsilon}(\nu(t)) & =\frac{1}{2} I_{\varepsilon}^{\prime}(\nu(t)) \nu(t)+\int \widehat{F}\left(\varepsilon x,\left|\nu(t)+h_{\varepsilon}(\nu(t))\right|\right) \\
& \geq \frac{1}{2} I_{\varepsilon}^{\prime}(\nu(t)) \nu(t)>0,
\end{aligned}
$$

and this contradicts the definition of $\Gamma_{\varepsilon}$. Consequently, by noting that $\nu(t)$ crosses $\mathscr{N}$ provided $\nu \in \Gamma_{\varepsilon}$, we have $d_{\varepsilon} \leq c_{\varepsilon}$.

Lemma 3.9. $c_{\varepsilon} \leq \gamma_{V_{0}}+o(1)$ as $\varepsilon \rightarrow 0$.

Proof. Again, let $w=w^{+}+w^{-} \in \mathscr{R}_{V_{0}}$, set $t_{0}>0$ such that $J_{V_{0}}\left(t_{0} w^{+}\right) \leq-1$. By virtue of Lemma 3.5 and Lemma 3.8, it sufficient to prove

$$
I_{\varepsilon}\left(t w^{+}\right)=J_{V_{0}}\left(t w^{+}\right)+o(1) \quad \text { uniformly in } t \in\left[0, t_{0}\right]
$$

as $\varepsilon \rightarrow 0$.

To this end, we only need to show the family $\left\{H_{\varepsilon}\right\} \subset C\left(\left[0, t_{0}\right]\right)$

$$
H_{\varepsilon}:\left[0, t_{0}\right] \rightarrow \mathbb{R}, \quad t \mapsto I_{\varepsilon}\left(t w^{+}\right)-J_{V_{0}}\left(t w^{+}\right)
$$

is equicontinuous. Observe that the boundedness of $h_{\varepsilon}$ and $\widetilde{\Phi}_{\varepsilon}^{\prime \prime}$ imply the boundedness of $h_{\varepsilon}^{\prime}$ due to (3.5) and (3.6), we conclude the derivatives of the family defined in (3.22) are uniformly bounded. Then the proof ends with a trivial application of Arzelà-Ascoli theorem.

\section{Proof of the main results}

We are now presenting the proof of the main results on the nonlinear Dirac equation:

$$
-i \alpha \cdot \nabla u+a \beta u+V_{\varepsilon}(x) u=g(|u|) u .
$$

Except for the hypotheses in Theorem 1.1 and Theorem 1.2, without loss of generality, we may assume that the boundary of $\Lambda$ is smooth, and that $0 \in \Lambda$ such that $V(0)=V_{0}:=\min _{\Lambda} V$. 
Thanks to the preparatory results already proved in Section 3, we will give an unified prove cover both super-linear and asymptotically linear cases. As mentioned before, in order to localize the desired solutions, we consider the modification of the function $g$ given by $f$ in (2.6) and the associated equation

$$
-i \alpha \cdot \nabla u+a \beta u+V_{\varepsilon}(x) u=f(\varepsilon x,|u|) u .
$$

For ease of notations, let us denote

$$
\mathscr{A}=\left\{x \in \Lambda: V(x)=V_{0}\right\} .
$$

And for the later use, letting $D=-i \alpha \cdot \nabla$, we rewrite (4.2) as

$$
D u=-a \beta u-V_{\varepsilon}(x) u+f(\varepsilon x,|u|) u .
$$

Acting the operator $D$ on the two sides of the above representation and noting that $D^{2}=-\Delta$, we find

$$
\Delta u=\left(a^{2}-V_{\varepsilon}^{2}(x)\right) u-f^{2}(\varepsilon x,|u|) u+D\left(V_{\varepsilon}(x)-f(\varepsilon x,|u|)\right) u .
$$

Letting

$$
\operatorname{sgn} u=\left\{\begin{array}{l}
\frac{\bar{u}}{|u|} \quad \text { if } u \neq 0, \\
0 \quad \text { if } u=0,
\end{array}\right.
$$

by the Kato's inequality [6], there holds

$$
\Delta|u| \geq \Re[\Delta u \cdot(\operatorname{sgn} u)] .
$$

Observe that

$$
\Re\left[D\left(V_{\varepsilon}(x)-f(\varepsilon x,|u|)\right) u \cdot \frac{\bar{u}}{|u|}\right]=0,
$$

hence

$$
\Delta|u| \geq\left(a^{2}-V_{\varepsilon}^{2}(x)\right)|u|-f^{2}(\varepsilon x,|u|)|u| .
$$

We remind that (4.3) together with the regularity results for $u$ (see Lemma 2.6) imply there is $M>0$ (independent of $\varepsilon$ ) satisfying

$$
\Delta|u| \geq-M|u|
$$

It then follows from the sub-solution estimate $[22,33]$ that

$$
|u(x)| \leq C_{0} \int_{B_{1}(x)}|u(y)| d y
$$

with $C_{0}>0$ independent of $x, \varepsilon$ and $u \in \mathscr{L}_{\varepsilon}$. 
Lemma 4.1. Assuming $\left(f_{1}\right)-\left(f_{6}\right)$ and, for all $\varepsilon$ sufficiently small, let $u_{\varepsilon} \in$ $\mathscr{L}_{\varepsilon}$, then $\left|u_{\varepsilon}\right|$ possesses a (global) maximum $x_{\varepsilon} \in \Lambda_{\varepsilon}$ such that

$$
\lim _{\varepsilon \rightarrow 0} V\left(\varepsilon x_{\varepsilon}\right)=V_{0}=\min _{x \in \Lambda} V(x) .
$$

Moreover, by setting $v_{\varepsilon}(x)=u_{\varepsilon}\left(x+x_{\varepsilon}\right)$, we must have $\left|v_{\varepsilon}\right|$ decays uniformly at infinity and $\left\{v_{\varepsilon}\right\}$ converges in $H^{1}$ to a ground state solution to

$$
-i \alpha \cdot \nabla v+a \beta v+V_{0} v=g(|v|) v .
$$

Proof. Let $u_{\varepsilon} \in E$ be the critical point so that $\widetilde{\Phi}_{\varepsilon}\left(u_{\varepsilon}\right)=c_{\varepsilon}$. We have $\left\{u_{\varepsilon}\right\}$ is a bounded set in $E$.

Step 1. $\left\{u_{\varepsilon}\right\}$ is non-vanishing.

Suppose contrarily that

$$
\sup _{x \in \mathbb{R}^{3}} \int_{B_{R}(x)}\left|u_{\varepsilon}\right|^{2} d x \rightarrow 0 \quad \text { as } \varepsilon \rightarrow 0
$$

for all $R>0$. Then, by Lion's concentration principle [25], $\left|u_{\varepsilon}\right|_{q} \rightarrow 0$ for $q \in(2,3)$. Since $\left\{u_{\varepsilon}\right\}$ is bounded in $E$, we have meas $\left\{x \in \mathbb{R}:\left|u_{\varepsilon}(x)\right| \geq r\right\}$ is uniformly bounded for all $\varepsilon>0$ provided $r>0$ is fixed. So we find

$$
\int_{\left\{x \in \mathbb{R}:\left|u_{\varepsilon}(x)\right| \geq r\right\}}\left|u_{\varepsilon}\right|^{2} d x \rightarrow 0
$$

as $\varepsilon \rightarrow 0$. Now, we have

$$
c_{\varepsilon}=\widetilde{\Phi}_{\varepsilon}\left(u_{\varepsilon}\right)-\frac{1}{2} \widetilde{\Phi}_{\varepsilon}^{\prime}\left(u_{\varepsilon}\right) u_{\varepsilon}=\int \widehat{F}\left(\varepsilon x,\left|u_{\varepsilon}\right|\right)=o(1),
$$

which contradict to the fact $c_{\varepsilon} \geq \tau>0$ (see Lemma 3.6).

Step 2. $\left\{\chi_{\Lambda_{\varepsilon}} \cdot u_{\varepsilon}\right\}$ is non-vanishing.

Indeed, if $\left\{\chi_{\Lambda_{\varepsilon}} \cdot u_{\varepsilon}\right\}$ vanishes, by virtue of Step 1 we have $\left\{\left(1-\chi_{\Lambda_{\varepsilon}}\right) \cdot u_{\varepsilon}\right\}$ is non-vanishing, that is (together with the fact that each $u_{\varepsilon}$ is decaying at infinity) there exist $x_{\varepsilon} \in \mathbb{R}^{3}$ and constants $R>0$ and $\delta>0$ such that $B_{R}\left(x_{\varepsilon}\right) \subset \mathbb{R}^{3} \backslash \Lambda_{\varepsilon}$ and

$$
\int_{B_{R}\left(x_{\varepsilon}\right)}\left|u_{\varepsilon}\right|^{2} \geq \delta
$$

Set $v_{\varepsilon}(x)=u_{\varepsilon}\left(x+x_{\varepsilon}\right)$, then $v_{\varepsilon}$ satifies

$$
-i \alpha \cdot \nabla v_{\varepsilon}+a \beta v_{\varepsilon}+\hat{V}_{\varepsilon}(x) v_{\varepsilon}=f\left(\varepsilon\left(x+x_{\varepsilon}\right),\left|v_{\varepsilon}\right|\right) v_{\varepsilon},
$$

where $\hat{V}_{\varepsilon}(x):=V\left(\varepsilon\left(x+x_{\varepsilon}\right)\right)$. Additionally, $v_{\varepsilon} \rightarrow v$ in $E$ and $v_{\varepsilon} \rightarrow v$ in $L_{l o c}^{q}$ for $q \in[1,3)$. Now assume without loss of generality that $V\left(\varepsilon x_{\varepsilon}\right) \rightarrow V_{\infty}$, using $\psi \in C_{c}^{\infty}\left(\mathbb{R}^{3}, \mathbb{C}^{4}\right)$ as a test function in (4.5), one gets

$$
\begin{aligned}
0 & =\lim _{\varepsilon \rightarrow 0} \int\left(-i \alpha \cdot \nabla v_{\varepsilon}+a \beta v_{\varepsilon}+\hat{V}_{\varepsilon}(x) v_{\varepsilon}-f\left(\varepsilon\left(x+x_{\varepsilon}\right),\left|v_{\varepsilon}\right|\right) v_{\varepsilon}\right) \bar{\psi} \\
& =\int\left(-i \alpha \cdot \nabla v+a \beta v+V_{\infty} v-\tilde{g}(|v|) v\right) \bar{\psi}
\end{aligned}
$$


Hence $v$ satisfies

$$
-i \alpha \cdot \nabla v+a \beta v+V_{\infty} v=\tilde{g}(|v|) v .
$$

However, using the test function $v^{+}-v^{-}$in (4.6), we have (with $\left(f_{3}\right)$ )

$$
\begin{aligned}
0 & =\|v\|^{2}+V_{\infty} \int v \cdot \overline{\left(v^{+}-v^{-}\right)}-\int \tilde{g}(|v|) v \cdot \overline{\left(v^{+}-v^{-}\right)} \\
& \geq\|v\|^{2}-\frac{|V|_{\infty}}{a}\|v\|^{2}-\frac{a-|V|_{\infty}}{2 a}\|v\|^{2} \\
& =\frac{a-|V|_{\infty}}{2 a}\|v\|^{2} .
\end{aligned}
$$

Therefore, we have $v=0$ a contradiction.

Step 3. Let $x_{\varepsilon} \in \mathbb{R}^{3}$ and $R, \delta>0$ be such that

$$
\int_{B_{R}\left(x_{\varepsilon}\right)}\left|\chi_{\Lambda_{\varepsilon}} \cdot u_{\varepsilon}\right|^{2} \geq \delta
$$

Then $\varepsilon x_{\varepsilon} \rightarrow \mathscr{A}$

To prove this, clearly, we may first choose $x_{\varepsilon} \in \Lambda_{\varepsilon}$, i.e. $\varepsilon x_{\varepsilon} \in \Lambda$. Suppose that, up to a subsequence if necessary, $\varepsilon x_{\varepsilon} \rightarrow x_{0} \in \bar{\Lambda}$ as $\varepsilon \rightarrow 0$. Again, set $v_{\varepsilon}(x)=u_{\varepsilon}\left(x+x_{\varepsilon}\right)$, we have $v_{\varepsilon} \rightarrow v$ in $E$ and $v$ satisfies

$$
-i \alpha \cdot \nabla v+a \beta v+V\left(x_{0}\right) v=f_{\infty}(x,|v|) v,
$$

where $f_{\infty}(x, s)=\chi_{\infty} \cdot g(s)+\left(1-\chi_{\infty}\right) \cdot \tilde{g}(s)$ and $\chi_{\infty}$ is either a characteristic function of a half-space of $\mathbb{R}^{3}$ provided

$$
\limsup _{\varepsilon \rightarrow 0} \operatorname{dist}\left(x_{\varepsilon}, \partial \Lambda_{\varepsilon}\right)<+\infty
$$

or $\chi_{\infty} \equiv 1$ ( ince $\Lambda$ is an open set with smooth boundary, this can be see by the fact $\chi_{\Lambda}\left(\varepsilon\left(\cdot+x_{\varepsilon}\right)\right)$ converges pointwise a.e. on $\mathbb{R}^{3}$ to $\chi_{\infty}(\cdot)$ and $\left.x_{\varepsilon} \in \Lambda_{\varepsilon}\right)$. Denote $S_{\infty}$ to be the associate energy functional to (4.7):

$$
S_{\infty}(u):=\frac{1}{2}\left(\left\|u^{+}\right\|^{2}-\left\|u^{-}\right\|^{2}\right)+\frac{V\left(x_{0}\right)}{2}|u|_{2}^{2}-\Psi_{\infty}(u),
$$

where

$$
\Psi_{\infty}(u):=\int F_{\infty}(x,|u|) \quad \text { and } \quad F_{\infty}(x, s)=\int_{0}^{s} f_{\infty}(x, \tau) \tau d \tau .
$$

By noting that $\Psi_{\infty}(u) \leq \Psi(u)$ (thanks to $\left(f_{2}\right)$ ), we have

$$
S_{\infty}(u) \geq \mathscr{T}_{V\left(x_{0}\right)}(u)=\mathscr{T}_{V_{0}}(u)+\frac{V\left(x_{0}\right)-V_{0}}{2}|u|_{2}^{2} \quad \text { for all } u \in E .
$$


Furthermore, if $s>0$ we find from $\left(g_{1}\right),\left(g_{3}\right)$ and the definition of $\tilde{g}$ that $\tilde{g}^{\prime}(s) s>0$. Hence, if $u \in E \backslash\{0\}$ and $v \in E$, we have

$$
\begin{aligned}
& \left(\Psi_{\infty}^{\prime \prime}(u)[u, u]-\Psi_{\infty}^{\prime}(u) u\right)+2\left(\Psi_{\infty}^{\prime \prime}(u)[u, v]-\Psi_{\infty}^{\prime}(u) v\right)+\Psi_{\infty}^{\prime \prime}(u)[v, v] \\
= & \int f_{\infty}(x,|u|)|v|^{2}+\int \partial_{s} f_{\infty}(x,|u|)|u|\left(|u|+\frac{\Re u \cdot v}{|u|}\right)^{2}>0 .
\end{aligned}
$$

Now let us define (as before) $h_{\infty}: E^{+} \rightarrow E^{-}$and $I_{\infty}: E^{+} \rightarrow \mathbb{R}$ by

$$
\begin{gathered}
S_{\infty}\left(u+h_{\infty}(u)\right)=\max _{v \in E^{-}} S_{\infty}(u+v), \\
I_{\infty}(u)=S_{\infty}\left(u+h_{\infty}(u)\right) .
\end{gathered}
$$

It is standard to see that: if $z \in E^{+} \backslash\{0\}$ satisfies $I_{\infty}^{\prime}(z) z=0$, then $I_{\infty}^{\prime \prime}(z)[z, z]<0$ (see [1]). Since we already have $v \neq 0$ is a critical point of $S_{\infty}$, we then infer $v^{+}$is a critical point of $I_{\infty}$ and $I_{\infty}\left(v^{+}\right)=\max _{t \geq 0} I_{\infty}\left(t v^{+}\right)$. Let $\tau>0$ such that $J_{V_{0}}\left(\tau v^{+}\right)=\max _{t \geq 0} J_{V_{0}}\left(t v^{+}\right)$, we infer

$$
\begin{aligned}
S_{\infty}(v) & =I_{\infty}\left(v^{+}\right)=\max _{t \geq 0} I_{\infty}\left(t v^{+}\right) \\
& \geq I_{\infty}\left(\tau v^{+}\right)=S_{\infty}\left(\tau v^{+}+h_{\infty}\left(\tau v^{+}\right)\right) \\
& \geq S_{\infty}\left(\tau v^{+}+\mathscr{J}_{V_{0}}\left(\tau v^{+}\right)\right) \\
& \geq \mathscr{T}_{V_{0}}\left(\tau v^{+}+\mathscr{J}_{V_{0}}\left(\tau v^{+}\right)\right)+\frac{V\left(x_{0}\right)-V_{0}}{2}\left|\tau v^{+}+\mathscr{J}_{V_{0}}\left(\tau v^{+}\right)\right|_{2}^{2} \\
& \geq \gamma_{V_{0}}+\frac{V\left(x_{0}\right)-V_{0}}{2}\left|\tau v^{+}+\mathscr{J}_{V_{0}}\left(\tau v^{+}\right)\right|_{2}^{2} .
\end{aligned}
$$

On the other hand, by Fatou's lemma, we deduce

$$
\begin{aligned}
c_{\varepsilon} & =\widetilde{\Phi}_{\varepsilon}\left(u_{\varepsilon}\right)-\frac{1}{2} \widetilde{\Phi}_{\varepsilon}^{\prime}\left(u_{\varepsilon}\right) u_{\varepsilon} \\
& =\int \widehat{F}\left(\varepsilon x,\left|u_{\varepsilon}\right|\right)=\int \widehat{F}\left(\varepsilon\left(x+x_{\varepsilon}\right),\left|v_{\varepsilon}\right|\right) \\
& \geq \int \widehat{F}_{\infty}(x,|v|) \\
& =S_{\infty}(v)-\frac{1}{2} S_{\infty}^{\prime}(v) v=S_{\infty}(v),
\end{aligned}
$$

where $\widehat{F}_{\infty}(x, s):=\frac{1}{2} f_{\infty}(x, s) s^{2}-F_{\infty}(x, s)$ for $(x, s) \in \mathbb{R}^{3} \times \mathbb{R}^{+}$. Therefore, together with (4.8), we have $c_{\varepsilon} \geq \gamma_{V_{0}}$ and $c_{\varepsilon}>\gamma_{V_{0}}$ provided $V\left(x_{0}\right) \neq V_{0}$. Therefore, by virtue of Lemma 3.9, we soon have $x_{0} \in \mathscr{A}$ and $\chi_{\infty} \equiv 1$.

Step 4. Let $v_{\varepsilon}$ be defined in Step 3, then $v_{\varepsilon} \rightarrow v$ in $E$.

It sufficient to prove that there is a subsequence $\left\{v_{\varepsilon_{j}}\right\}$ such that $v_{\varepsilon_{j}} \rightarrow v$ in $E$. Recall that, as the argument shows, $v$ is a ground state solution to

$$
-i \alpha \cdot \nabla v+a \beta v+V_{0} v=g(|v|) v,
$$


and

$$
\lim _{\varepsilon \rightarrow 0} \int \widehat{F}\left(\varepsilon\left(x+x_{\varepsilon}\right),\left|v_{\varepsilon}\right|\right)=\int \widehat{G}(|v|) .
$$

Let $\eta:[0, \infty) \rightarrow[0,1]$ be a smooth function satisfying $\eta(s)=1$ if $s \leq 1$, $\eta(s)=0$ if $s \geq 2$. Define $\tilde{v}_{j}(x)=\eta(2|x| / j) v(x)$. One has

$$
\left\|\tilde{v}_{j}-v\right\| \rightarrow 0 \quad \text { and } \quad\left|\tilde{v}_{j}-v\right|_{q} \rightarrow 0 \quad \text { as } j \rightarrow \infty
$$

for $q \in[2,3]$. Set $B_{d}:=\left\{x \in \mathbb{R}^{3}:|x| \leq d\right\}$ for $d>0$. We have that there possesses a subsequence $\left\{v_{\varepsilon_{j}}\right\}$ such that, for any $\delta>0$ there exists $r_{\delta}>0$ satisfying

$$
\limsup _{j \rightarrow \infty} \int_{B_{j} \backslash B_{r}}\left|v_{\varepsilon_{j}}\right|^{q} \leq \delta
$$

for all $r \geq r_{\delta}$ (see an argument of [9, Lemma 5.7]). Here we will use

$$
q= \begin{cases}p & \text { for the super-linear case } \\ 2 & \text { for the asymptotically linear case }\end{cases}
$$

where $p \in(2,3)$ is the constant in condition $\left(g_{2}\right)(i)$. Denote $z_{j}=v_{\varepsilon_{j}}-\tilde{v}_{j}$, we remark that $\left\{z_{j}\right\}$ is bounded in $E$ and

$$
\begin{aligned}
\lim _{j \rightarrow \infty} \mid \int F\left(\varepsilon_{j}\left(x+x_{\varepsilon_{j}}\right),\left|v_{\varepsilon_{j}}\right|\right)-F\left(\varepsilon_{j}\left(x+x_{\varepsilon_{j}}\right),\left|z_{j}\right|\right) \\
-F\left(\varepsilon_{j}\left(x+x_{\varepsilon_{j}}\right),\left|\tilde{v}_{j}\right|\right) \mid=0
\end{aligned}
$$

and

$$
\begin{aligned}
\lim _{j \rightarrow \infty} \mid \int\left[f\left(\varepsilon_{j}\left(x+x_{\varepsilon_{j}}\right),\left|v_{\varepsilon_{j}}\right|\right) v_{\varepsilon_{j}}-f\left(\varepsilon_{j}\left(x+x_{\varepsilon_{j}}\right),\left|z_{j}\right|\right) z_{j}\right. \\
\left.-f\left(\varepsilon_{j}\left(x+x_{\varepsilon_{j}}\right),\left|\tilde{v}_{j}\right|\right) \tilde{v}_{j}\right] \bar{\varphi} \mid=0
\end{aligned}
$$

uniformly in $\varphi \in E$ with $\|\varphi\| \leq 1$ (see [9, Lemma 7.10]). Using the decay of $v$ and the fact that $\hat{V}_{\varepsilon_{j}}(x) \rightarrow V_{0}, F\left(\varepsilon_{j}\left(x+x_{\varepsilon_{j}}\right), s\right) \rightarrow G(s)$ as $j \rightarrow \infty$ uniformly on any bounded set of $x$, one checks easily the following

$$
\Re \int \hat{V}_{\varepsilon_{j}}(x) v_{\varepsilon_{j}} \cdot \tilde{v}_{j} \rightarrow \int V_{0} \cdot|v|^{2}, \quad \int F\left(\varepsilon_{j}\left(x+x_{\varepsilon_{j}}\right),\left|\tilde{v}_{j}\right|\right) \rightarrow \int G(|v|) .
$$

Let us denote $\hat{\Phi}_{\varepsilon}$ to be the associate energy functional of (4.5), then we have

$$
\begin{aligned}
\hat{\Phi}_{\varepsilon_{j}}\left(z_{j}\right)= & \hat{\Phi}_{\varepsilon_{j}}\left(v_{\varepsilon_{j}}\right)-S_{\infty}(v) \\
& +\int F\left(\varepsilon_{j}\left(x+x_{\varepsilon_{j}}\right),\left|v_{\varepsilon_{j}}\right|\right)-F\left(\varepsilon_{j}\left(x+x_{\varepsilon_{j}}\right),\left|z_{j}\right|\right) \\
& -F\left(\varepsilon_{j}\left(x+x_{\varepsilon_{j}}\right),\left|\tilde{v}_{j}\right|\right)+o(1) \\
= & o(1)
\end{aligned}
$$


as $j \rightarrow \infty$, which implies that $\hat{\Phi}_{\varepsilon_{j}}\left(z_{j}\right) \rightarrow 0$. Similarly,

$$
\begin{aligned}
\hat{\Phi}_{\varepsilon_{j}}^{\prime}\left(z_{j}\right) \varphi= & \Re \int\left[f\left(\varepsilon_{j}\left(x+x_{\varepsilon_{j}}\right),\left|v_{\varepsilon_{j}}\right|\right) v_{\varepsilon_{j}}-f\left(\varepsilon_{j}\left(x+x_{\varepsilon_{j}}\right),\left|z_{j}\right|\right) z_{j}\right. \\
& \left.-f\left(\varepsilon_{j}\left(x+x_{\varepsilon_{j}}\right),\left|\tilde{v}_{j}\right|\right) \tilde{v}_{j}\right] \bar{\varphi}+o(1) \\
= & o(1)
\end{aligned}
$$

as $j \rightarrow \infty$ uniformly in $\|\varphi\| \leq 1$, which implies $\hat{\Phi}_{\varepsilon_{j}}^{\prime}\left(z_{j}\right) \rightarrow 0$. Therefore,

$$
o(1)=\hat{\Phi}_{\varepsilon_{j}}\left(z_{j}\right)-\frac{1}{2} \hat{\Phi}_{\varepsilon_{j}}^{\prime}\left(z_{j}\right) z_{j}=\int \widehat{F}\left(\varepsilon_{j}\left(x+x_{\varepsilon_{j}}\right),\left|z_{j}\right|\right) .
$$

Owning to $\left(f_{6}\right)$ and the regularity result, for any fixed $r>0$, one has

$$
\int \widehat{F}\left(\varepsilon_{j}\left(x+x_{\varepsilon_{j}}\right),\left|z_{j}\right|\right) \geq C_{r} \int_{\left\{x \in \mathbb{R}^{3}:\left|z_{j}(x)\right| \geq r\right\}}\left|z_{j}\right|^{2}
$$

for some constant $C_{r}$ depends only on $r$. Hence

$$
\int_{\left\{x \in \mathbb{R}^{3}:\left|z_{j}(x)\right| \geq r\right\}}\left|z_{j}\right|^{2} \rightarrow 0
$$

as $j \rightarrow \infty$ for any fixed $r>0$. Notice $\left\{\left|z_{j}\right|_{\infty}\right\}$ is bounded, as a consequence, we get

$$
\begin{aligned}
\left(1-\frac{|V|_{\infty}}{a}\right)\left\|z_{j}\right\|^{2} \leq & \left\|z_{j}\right\|^{2}+\Re \int \hat{V}_{\varepsilon_{j}}(x) z_{j} \cdot \overline{\left(z_{j}^{+}-z_{j}^{-}\right)} \\
= & \hat{\Phi}_{\varepsilon_{j}}^{\prime}\left(z_{j}\right)\left(z_{j}^{+}-z_{j}^{-}\right) \\
& +\Re \int f\left(\varepsilon_{j}\left(x+x_{\varepsilon_{j}}\right),\left|z_{j}\right|\right) z_{j} \cdot \overline{\left(z_{j}^{+}-z_{j}^{-}\right)} \\
\leq & o(1)+\frac{a-|V|_{\infty}}{2 a}\left\|z_{j}\right\|^{2} \\
& +C_{\infty} \int_{\left\{x \in \mathbb{R}^{3}:\left|z_{j}(x)\right| \geq r\right\}}\left|z_{j}\right| \cdot\left|z_{j}^{+}-z_{j}^{-}\right| \\
\leq & o(1)+\frac{a-|V|_{\infty}}{2 a}\left\|z_{j}\right\|^{2},
\end{aligned}
$$

that is, $\left\|z_{j}\right\| \rightarrow 0$ as $j \rightarrow \infty$. Together with (4.10) we get $v_{\varepsilon_{j}} \rightarrow v$ in $E$.

Step 5. $v_{\varepsilon}(x) \rightarrow 0$ as $|x| \rightarrow \infty$ uniformly for all small $\varepsilon$.

Assume by contradiction that there exist $\delta>0$ and $y_{\varepsilon} \in \mathbb{R}^{3}$ with $\left|y_{\varepsilon}\right| \rightarrow$ $\infty$ such that

$$
\delta \leq\left|v_{\varepsilon}\left(y_{\varepsilon}\right)\right| \leq C_{0} \int_{B_{1}\left(y_{\varepsilon}\right)}\left|v_{\varepsilon}(y)\right| d y .
$$


Since $v_{\varepsilon} \rightarrow v$ in $E$, we obtain, as $\varepsilon \rightarrow 0$,

$$
\begin{aligned}
\delta & \leq C_{0}\left(\int_{B_{1}\left(y_{\varepsilon}\right)}\left|v_{\varepsilon}\right|^{2}\right)^{1 / 2} \\
& \leq C_{0}\left(\int\left|v_{\varepsilon}-v\right|^{2}\right)^{1 / 2}+C_{0}\left(\int_{B_{1}\left(y_{\varepsilon}\right)}|v|^{2}\right)^{1 / 2} \rightarrow 0,
\end{aligned}
$$

a contradiction.

By virtue of Step 5 it is clear that one may assume the sequence $\left\{x_{\varepsilon}\right\}$ in Step 3 to be the maximum points of $\left|u_{\varepsilon}\right|$. Moreover, from the above argument we readily see that, any sequence of such points satisfies $\varepsilon x_{\varepsilon}$ converging to some point in $\mathscr{A}$ as $\varepsilon \rightarrow 0$.

Finally, in order to verify that $v_{\varepsilon} \rightarrow v$ in $H^{1}$, we first deduce from (4.5) and (4.9) that

$$
H_{0}\left(v_{\varepsilon}-v\right)=f\left(\varepsilon\left(x+x_{\varepsilon}\right),\left|v_{j}\right|\right) v_{j}-g(|v|) v-\left(\hat{V}_{\varepsilon}(x) v_{j}-V\left(x_{0}\right) v\right) .
$$

Using Step 4 and the uniform estimate in Remark 2.7, it is easy to check that $\left|H_{0}\left(v_{\varepsilon}-v\right)\right|_{2} \rightarrow 0$ as $\varepsilon \rightarrow 0$. Therefore $v_{j} \rightarrow v$ in $H^{1}\left(\mathbb{R}^{3}, \mathbb{C}^{4}\right)$, ending the proof.

The Step 5 in the previous lemma shows an uniform decay estimate, not surprisingly, the decay rate can be shown to be exponential:

Lemma 4.2. There exist $C>0$ such that for all $\varepsilon>0$ small

$$
\left|u_{\varepsilon}(x)\right| \leq C e^{-c_{0}\left|x-x_{\varepsilon}\right|}
$$

where $c_{0}=\sqrt{\left(a^{2}-|V|_{\infty}^{2}\right)}$.

Proof. The uniform decay estimate together with (4.3) allow us to take $R>0$ sufficiently large such that

$$
\Delta\left|v_{\varepsilon}\right| \geq\left(a^{2}-|V|_{\infty}^{2}\right)\left|v_{\varepsilon}\right|
$$

for all $|x| \geq R$ and $\varepsilon>0$ small. Let $\Gamma(y)=\Gamma(y, 0)$ be a fundamental solution to $-\Delta+\left(a^{2}-|V|_{\infty}^{2}\right)$. Using the uniform boundedness, we may choose that $\left|v_{\varepsilon}(y)\right| \leq\left(a^{2}-|V|_{\infty}^{2}\right) \Gamma(y)$ holds on $|y|=R$ for all $\varepsilon>0$ small. Let $z_{\varepsilon}=\left|v_{\varepsilon}\right|-\left(a^{2}-|V|_{\infty}^{2}\right) \Gamma$. Then

$$
\begin{aligned}
\Delta z_{\varepsilon} & =\Delta\left|v_{\varepsilon}\right|-\left(a^{2}-|V|_{\infty}^{2}\right) \Delta \Gamma \\
& \geq\left(a^{2}-|V|_{\infty}^{2}\right)\left(\left|v_{\varepsilon}\right|-\left(a^{2}-|V|_{\infty}^{2}\right) \Gamma\right)=\left(a^{2}-|V|_{\infty}^{2}\right) z_{\varepsilon} .
\end{aligned}
$$

By the maximum principle we can conclude that $z_{\varepsilon}(y) \leq 0$ on $|y| \geq R$. It is well known that there is $C^{\prime}>0$ such that $\Gamma(y) \leq C^{\prime} \exp \left(-c_{0}|y|\right)$ on $|y| \geq 1$, we see that

$$
\left|v_{\varepsilon}(y)\right| \leq C^{\prime \prime} e^{-c_{0}|y|}
$$


for all $y \in \mathbb{R}^{3}$ and all $\varepsilon>0$ small, that is

$$
\left|u_{\varepsilon}(x)\right| \leq C e^{-c_{0}\left|x-x_{\varepsilon}\right|}
$$

as claimed.

Now, we are ready to prove our main theorems.

Unified proof of Theorem 1.1 and Theorem 1.2. Define

$$
w_{\varepsilon}(x)=u_{\varepsilon}(x / \varepsilon) \quad \text { and } \quad y_{\varepsilon}=\varepsilon x_{\varepsilon} .
$$

Then $w_{\varepsilon}$ is a solution of

$$
-i \varepsilon \alpha \cdot \nabla w+a \beta w+V(x) w=f(x,|w|) w
$$

for all $\varepsilon>0$. Since $y_{\varepsilon}$ is a maximum point of $\left|w_{\varepsilon}\right|$, due to Lemma 4.1 and Lemma 4.2, we have

$$
\left|w_{\varepsilon}(x)\right| \leq C e^{-\frac{c_{0}}{\varepsilon}\left|x-y_{\varepsilon}\right|}
$$

and $y_{\varepsilon} \rightarrow \mathscr{A}$ as $\varepsilon \rightarrow 0$. From the assumption

$$
\min _{\Lambda} V<\min _{\partial \Lambda} V
$$

we conclude that $\delta:=\operatorname{dist}(\mathscr{A}, \partial \Lambda)>0$. Hence, for $\varepsilon$ sufficiently small, one find actually $\left|w_{\varepsilon}(x)\right| \leq C \exp \left(-\frac{c_{0} \delta}{2 \varepsilon}\right)<\xi$ if $x \notin \Lambda$. Therefore, $f\left(x,\left|w_{\varepsilon}\right|\right)=$ $g\left(\left|w_{\varepsilon}\right|\right)$ for all $\varepsilon>0$ small enough, and the proof of the theorems is thereby completed.

Acknowledgment. The authors would like to thank the anonymous reviewer for his/her helpful comments. The work was supported by the National Science Foundation of China (NSFC11331010, 11171286) and the Beijing Center for Mathematics and Information Interdisciplinary Sciences.

\section{References}

[1] N. Ackermann, A nonlinear superposition principle and multibump solution of periodic Schrödinger equations, J. Funct. Anal. 234 (2006) 423-443.

[2] A. Ambrosetti, M. Badiale, S. Cignolani, Semi-classical states of nonlinear Shrödinger equations, Arch. Rational Mech. Anal. 140 (1997), 285-300.

[3] A. Ambrosetti, V. Felli, A. Malchiodi, Ground states of nonlinear Schrödinger equations with potentials vanishing at infinity, J. Eur. Math. Soc. 7 (2005), 117-144. 
[4] J. Byeon, L. Jeanjean, Standing waves for nonlinear Schrödinger equations with a general nonlinearity, Arch. Rational Mech. Anal. 185.2 (2007): 185-200.

[5] J. Byeon, Z.Q. Wang, Standing waves with a critical frequency for nonlinear Schrödinger equations, Arch. Rational Mech. Anal. 165.4 (2002): 295-316.

[6] R. Dautray, J.L. Lions, Mathematical Analysis and Numerical Methods for Science and Technology, vol. 3, Springer, Berlin, 1990.

[7] M. Del Pino, P. Felmer, Local mountain passes for semilinear ellipitc problems in unbounded domains, Calc. Var. Partial Differential Equations 4 (1996), 121-137.

[8] M. Del Pino, P. Felmer, Multi-peak bound states for nonlinear Schrödinger equations, Annales de l'Institut Henri Poincare (C) Non Linear Analysis. Vol. 15. No. 2. Elsevier Masson, 1998, 127-149.

[9] Y.H. Ding, Variational Methods for Strongly Indefinite Problems, Interdiscip. Math. Sci., 7, World Scientific Publ., 2007.

[10] Y.H. Ding, Semi-classical ground states concentrating on the nonlinear potentical for a Dirac equation, J. Differential Equations 249 (2010) 1015-1034.

[11] Y.H. Ding, C. Lee, B. Ruf, On semiclassical states of a nonlinear Dirac equation, Proceedings of the Royal Society of Edinburgh: Section A Mathematics 143.04 (2013): 765-790.

[12] Y.H. Ding, Xiaoying Liu, Semi-classical limits of ground states of a nonlinear Dirac equation, J. Differential Equations 252 (2012) 49624987.

[13] Y.H. Ding and B. Ruf, Existence and concentration of semi-classical solutions for Dirac equations with critical nonlinearities, SIAM Journal on Mathematical Analysis 44.6 (2012): 3755-3785.

[14] Y.H. Ding, J.C. Wei, Stationary states of nonlinear Dirac equations with general potentials, Rev. Math. Phys. 20 (2008) 1007-1032.

[15] Y.H. Ding, J.C. Wei and T. Xu, Existence and concentration of semiclassical solutions for a nonlinear Maxwell-Dirac system, J. Math. Phys. 54 (2013), no. 06, 061505, 33 pp.

[16] Y.H. Ding, T. Xu, On semi-classical limits of ground states of a nonlinear Maxwell-Dirac system, Calc. Var. Partial Differential Equations (2014) 51(1): 17-44. 
[17] Y.H. Ding, T. Xu, On the concentration of semi-classical states for a nonlinear Dirac-Klein-Gordon system, J. Differential Equations 256 (2014) 1264-1294.

[18] Maria J. Esteban and Eric Séré, Stationary states of the nonlinear Dirac equation: A variational approach, Comm. Math. Phys. 171 (1995) 323-350.

[19] R. Finkelstein, R. LeLevier, M. Ruderman, Nonlinear spinor fields, Physical Review 83.2 (1951): 326-332.

[20] R. Finkelstein, C. Fronsdal, P. Kaus, Nonlinear spinor field, Physical Review 103.5 (1956): 1571-1579.

[21] A. Floer, A. Weinstein, Nonspreading wave packets for the cubic Schrödinger equation with a bounded potential, J. Funct. Anal. 69 (1986), 397-408.

[22] D. Gilbarg, N.S. Trudinger, Elliptic Partial Differential Equations of Second Order, Springer, Berlin, 1998.

[23] D.D. Ivanenko, Notes to the theory of interaction via particles, Zh.Éksp. Teor. Fiz. 8 (1938) 260-266.

[24] L. Jeanjean, K. Tanaka, Singularly perturbed elliptic problems with superlinear or asymptotically linear nonlinearities, Calc. Var. Partial Differential Equations (2004) 21(3): 287-318.

[25] P.L. Lions, The concentration-compactness principle in the calculus of variations: The locally compact case, Part II, AIP Anal. non linéaire $1,223-283$.

[26] R. Miguel, T. Hugo, Solutions with multiple spike patterns for an elliptic system. Calc. Var. Partial Differential Equations (2008) 31(1), $1-25$.

[27] R. Miguel, J.F. Yang, Spike-layered solutions for an elliptic system with Neumann boundary conditions, Trans. Amer. Math. Soc. 357 (2005) 3265-3284.

[28] Y.G. Oh, Existence of semi-classical bound states of nonlinear Schrödinger equations with potentials of the class $(V)_{a}$, Comm. Partial Differential Equations 13.12 (1988): 1499-1519.

[29] Y.G. Oh, On positive multi-lump bound states of nonlinear Schrödinger equations under multiple well potential, Comm. Math. Phys. 131.2 (1990): 223-253. 
[30] P.H. Rabinowitz, Minimax Methods in Critical Point Theory with Applications to Differential Equations, Amer. Math. Soc., Providence, 1986.

[31] P.H. Rabinowitz, On a class of nonlinear Schrödinger equations, Z. Angew Math Phys 43.2 (1992): 270-291.

[32] E.M. Stein, Singular integrals and differentiability properties of functions, Vol. 2. Princeton university press, 1970.

[33] B. Simon, Schrödinger semigroups, Bull. Amer. Math. Soc. (N.S.) 7 (1982) 447-526.

[34] X. Wang, On concentration of positive bound states of nonlinear Schrödinger equations, Comm. Math. Phys. 153.2 (1993): 229-244. 